\title{
A DNA barcode library for the water mites of
}

\section{Montenegro}

\author{
Vladimir Pešić ${ }^{\ddagger}$, Andrzej Zawal§ , Ana Manovićł, Aleksandra Bańkowskal, Milica Jovanović ${ }^{\ddagger}$ \\ $\ddagger$ Department of Biology, University of Montenegro, Podgorica, Montenegro \\ $\S$ Institute of Marine and Environmental Sciences, Center of Molecular Biology and Biotechnology, \\ University of Szczecin, Szczecin, Poland \\ | Institute of Biology, University of Szczecin, Szczecin, Poland
}

Corresponding author: Vladimir Pešić (vladopesic@gmail.com)

Academic editor: Fernando Jacinavicius

Received: 22 Nov 2021 | Accepted: 16 Dec 2021 | Published: 20 Dec 2021

Citation: Pešić V, Zawal A, Manović A, Bańkowska A, Jovanović M (2021) A DNA barcode library for the water mites of Montenegro. Biodiversity Data Journal 9: e78311. https://doi.org/10.3897/BDJ.9.e78311

\begin{abstract}
Water mites (Acari, Hydrachnidia) are a significant component of freshwater ecosystems inhabiting a wide range of aquatic habitats. This study provides a first comprehensive DNA barcode library for the water mites of Montenegro. DNA barcodes were analysed from 233 specimens of water mites morphologically assigned to 86 species from 28 genera and 15 families. In the course of the study, four species, i.e. Lebertia reticulata (Koenike, 1919), Atractides inflatipalpis K.Viets, 1950, A. latipes (Szalay, 1935) and Parabrachypoda montii (Maglio, 1924) were molecularly confirmed as new for Montenegro and three species, i.e. Protzia octopora Lundblad, 1954, Piona laminata (Thor, 1901) and Unionicola ypsilophora (Bonz, 1783) are new for the Balkan Peninsula. Results are analysed using the Barcode Index Number system (BIN) and the Refined Single Linkage (RESL) of BOLD. The BIN assigned sequences to 98 clusters, while the RESL reveal 103 operational taxonomic units (OTUs). Unique BINs were revealed for 72 species $(83.7 \%)$, whereas twelve species $(14 \%)$ were characterised by two BINs and two species $(2.3 \%)$ with three BINs. Amongst the studied taxa, 14 species were found with a high intraspecific sequence divergences (' $2.2 \%$ ), emphasising the need for additional comprehensive morphological and molecular analysis of these species.
\end{abstract}




\section{Keywords}

DNA barcoding, COI, water mites, Montenegro, species delimitation

\section{Introduction}

Hydrachnidia, also known as water mites, is a most diverse and abundant group of arachnids in freshwater habitats (Davids et al. 2007). With nearly 7,500 species grouped into 550 genera (Smit 2020), they inhabit a wide range of aquatic habitats, including lotic, lentic, interstitial and temporary waters. Water mites have a complex life cycle that includes two pupa-like resting stages, i.e. protonymph and tritonymph and three active stages: larva is almost always parasitic, deutonymphs and adults that are predators of minute invertebrates (Davids et al. 2007). Some recent studies have shown that water mites can be good indicators of ecosystem health, especially of groundwater-dependent ecosystems (Pešić et al. 2019b). However, their time-consuming taxonomic identification has been identified as a major constraint for more significant involvement in rapid assessment programmes (Weigand et al. 2019).

Traditional morphology often underestimates the true diversity of water mites and, in recent years, it has been successfully replaced by an integrative approach that combines both morphological characteristics and molecular data (Martin et al. 2010, Pešić et al. 2017, Fisher et al. 2017, Pešić et al. 2019a, Pešić et al. 2020d, Pešić and Smit 2020). This process has been enhanced by the formation of the comprehensive DNA barcode reference libraries, such as the BOLD System (https://www.boldsystems.org/) and GenBank (https://www.ncbi.nlm.nih.gov/). DNA barcodes have been proposed and successfully adopted for water mites as an efficient method for detecting previously overlooked and/or misidentified species (Martin et al. 2010, Pešić et al. 2017, Pešić et al. 2019). The significant increase in the number of studies using DNA barcodes in recent years, especially in some regions, has laid the foundations for building a comprehensive library of DNA barcodes at the national and/or regional level (e.g. Blattner et al. 2019).

Of the Balkan countries, Montenegro is one of the best studied from the taxonomic and faunistic point of view (Pešić et al. 2018). Water mite research began in 1903 when the Czech zoologist Karl Thon published the first list of 13 species (Thon 1903). For more than one century, a large number of papers on the Montenegrin water mites have been published (Musselius 1912, Viets 1936, Pešić 2001, Pešić 2002b, Pešić 2002d, Pešić 2002a, Pešić 2002c, Pešić 2003a, Pešić 2003c, Pešić and Gerecke 2003, Di Sabatino et al. 2003, Pešić 2003b, Pešić 2004b, Pešić 2004a, Smit and Pešić 2004, Baker et al. 2008, Pešić et al. 2010, Pešić et al. 2012, Pešić et al. 2017, Bańkowska et al. 2016, Pešić et al. 2018, Zawal and Pešić 2018, Pešić et al. 2019a, Pešić et al. 2019c, Pešić et al. 2020a, Pešić et al. 2020b, Pešić et al. 2020c, Pešić et al. 2020d, Zawal et al. 2020, Pešić and Smit 2020, Pešić et al. 2021b)

Currently, 201 species of water mites have been reported for Montenegro (Pešić et al. 2018, Pešić et al. 2019c, Pešić et al. 2020c, Pešić et al. 2020d, Pešić and Smit 2020). This 
number makes up about $50 \%$ of the species known from the Balkans, which is estimated at about 400 species (Pešić et al. 2018). This is still a small number for the area of such hydrogeological characteristics and the turbulent geological history as the Balkans. Therefore, there is no reason not to believe that the expected number of water mites in the Balkans is at least at the level of Central Europe which is home to approximately 745 species (Gerecke et al. 2016).

The aim of the study is to develop and evaluate the first library of barcodes for water mites from Montenegro, targeting a COI fragment of $\sim 658 \mathrm{bp}$. Taking advantage of publicly available DNA barcode reference libraries, such as the BOLD and the use of the universal Barcode Index Number (BIN), allows us to assess the molecular diversity of water mite species inhabiting the territory of Montenegro, as well as to explore their distribution patterns in Europe. Moreover, this approach will allow us to also identify problematic species groups both for traditional taxonomy and for DNA barcoding.

\section{Material and methods}

Water mites were collected by hand netting, sorted live in the field and immediately preserved in $96 \%$ ethanol (EtOH) for the molecular analysis. Water mites were collected from 54 sampling sites in Montenegro (Fig. 1) during several sampling campaigns from 2018-2020. Photos from each studied specimen were taken before molecular work started. The photographs were made using a camera on a Samsung Galaxy smartphone.

Molecular analysis were conducted in the Canadian Centre for DNA Barcoding (Guelph, Ontario, Canada; (CCDB; http://ccdb.ca/) and in the Department of Invertebrate Zoology and Hydrobiology (DIZH), University of Łódź, Poland. For the methods used for cytochrome $c$ oxidase subunit I (COI) gene amplification in DIZH, see Pešić et al. (2017). In $\mathrm{CCDB}$, the specimens were sequenced for the barcode region of $\mathrm{COI}$ using standard invertebrate DNA extraction (Ivanova et al. 2007), amplification (Ivanova and Grainger 2007a) and sequencing protocols (Ivanova and Grainger 2007b). The DNA extracts were archived in $-80^{\circ} \mathrm{C}$ freezers at the Centre for Biodiversity Genomics (CBG; biodiversitygenomics.net) and the specimen vouchers were stored in $95 \% \mathrm{EtOH}$ and returned to the first author for morphological examination. Some of these vouchers were dissected as described elsewhere (Davids et al. 2007) and slide-mounted in Faure's medium, while the rest were transferred to Koenike's medium and stored in the collection of the first author at the Department of Biology in Podgorica.

\section{DNA barcode analysis}

In CCDB, the chromatograms were assembled into consensus sequences for each specimen and uploaded to BOLD. The taxonomic account, voucher specimen ID, collecting locality and voucher depositor were incorporated into the system for further analysis. Water mite sequences, obtained during this study, were grouped in the "MNHYD" (DNA barcode reference library of Montenegrin water mites) dataset. Detailed voucher information, taxonomic classifications, photos, DNA barcode sequences, primer pairs used and trace 
files (including their quality) were uploaded to the dataset "MNHYD" on the Barcode of Life Data Systems (BOLD; www.boldsystems.org).

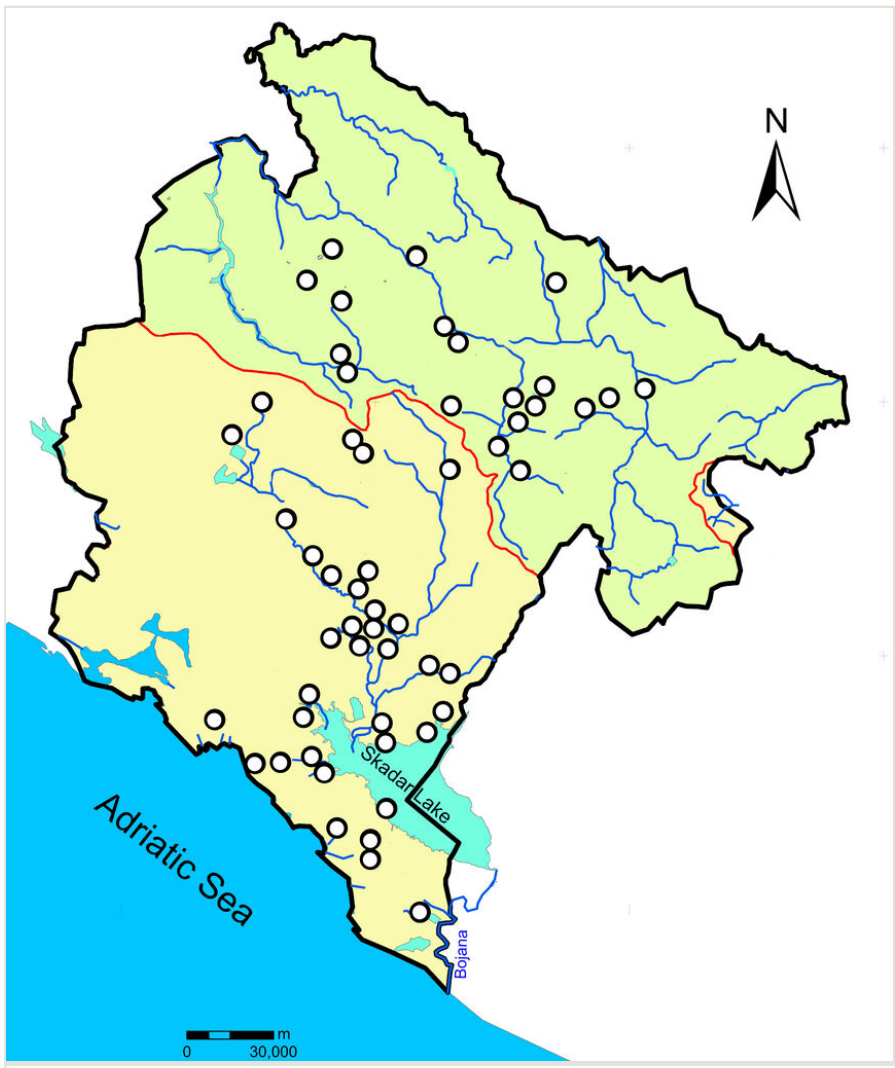

Figure 1. doi

Sampling sites from Montenegro. The green colour represents the Danube Basin (Black Sea) and the yellow colour represents the Adriatic Basin.

The translation of the $\mathrm{CO}$ sequences into amino acids did not contain any stop codon positions and blasting the sequences confirmed the absence of contaminations. In cases of the four Unionicola ypsylophora mites, we amplified Anodonta exulcerata DNA instead of water mite DNA. These specimens were excluded from further analysis.

The reference library for the molecular identification of water mites sequenced in this study was analysed using the Barcode Index Number system (BIN) (Felsenstein 1985). The distribution of BINs was performed by the Barcode of Life Data System v.4 (accessed 15 November 2021). The two-phase BIN analysis system in the first phase applies a first threshold of $2.2 \%$ (that allows a rough differentiation between intraspecific and interspecific distances), followed by refinements through Markov clustering into the final BINs (Ratnasingham and Hebert 2013). BOLD ID and accession numbers for all specimens included in final dataset are given in Table 1. 
Table 1.

Details on barcoded specimens from Montenegro.

\begin{tabular}{|c|c|c|c|c|c|}
\hline Taxa & Voucher Code & $\begin{array}{l}\text { BOLD } \\
\text { Process } \\
\text { ID }\end{array}$ & BIN & Locality & Coordinates \\
\hline \multicolumn{6}{|l|}{ Limnocharidae } \\
\hline $\begin{array}{l}\text { Limnochares } \\
\text { aquatica }\end{array}$ & $\begin{array}{l}31 . \\
\text { CG2020 } 6 \quad \text { C10 }\end{array}$ & DNAEC032-20 & BOLD:ACS0438 & $\begin{array}{l}\text { Podgorica, Zeta } \\
\text { River at Pričelje }\end{array}$ & $\begin{array}{l}42.5022 \mathrm{~N} \\
19.2225 \mathrm{E}\end{array}$ \\
\hline \multicolumn{6}{|l|}{ Hydryphantidae } \\
\hline \multirow[t]{3}{*}{ Panisus michaeli } & CCDB $38361 \mathrm{~A} 04$ & DCDDJ004-21 & \multirow[t]{3}{*}{ BOLD:ADT7504 } & $\begin{array}{l}\text { Kolašin, Lalevića } \\
\text { Dolovi, spring \#1 }\end{array}$ & $\begin{array}{l}42.899 \mathrm{~N} \\
19.631 \mathrm{E}\end{array}$ \\
\hline & $\underline{\text { CCDB } 38361 \mathrm{~A} 05}$ & DCDDJ005-21 & & $\begin{array}{l}\text { Kolašin, Lalevića } \\
\text { Dolovi, spring \#1 }\end{array}$ & $\begin{array}{l}42.899 \mathrm{~N} \\
19.631 \mathrm{E}\end{array}$ \\
\hline & CCDB $38361 \mathrm{~A} 06$ & DCDDJ006-21 & & $\begin{array}{l}\text { Kolašin, Lalevića } \\
\text { Dolovi, spring \#1 }\end{array}$ & $\begin{array}{l}42.899 \mathrm{~N} \\
19.631 \mathrm{E}\end{array}$ \\
\hline $\begin{array}{l}\text { Trichothyas } \\
\text { jadrankae }\end{array}$ & $\underline{\text { CCDB-38679-A08 }}$ & DNCBD008-20 & BOLD:AEF1286 & $\begin{array}{l}\text { Bar, Poseljanski } \\
\text { stream at Poseljani }\end{array}$ & $\begin{array}{l}42.3095 \mathrm{~N} \\
19.0518 \mathrm{E}\end{array}$ \\
\hline Partnunia naprintua & $\underline{\mathrm{CCDB} 38361 \mathrm{~A} 08}$ & DCDDJ008-21 & BOLD:AEL6734 & $\begin{array}{l}\text { Andrijevica, spring } \\
\text { at Trešnjevik }\end{array}$ & $\begin{array}{l}42.7392 \mathrm{~N} \\
19.6933 \mathrm{E}\end{array}$ \\
\hline \multirow[t]{4}{*}{ Protzia invalvaris } & CCDB 38361 C11 & DCDDJ035-21 & \multirow[t]{4}{*}{ BOLD:AEI2833 } & $\begin{array}{l}\text { Kolašin, Bistrica } \\
\text { stream }\end{array}$ & $\begin{array}{l}42.8054 \mathrm{~N} \\
19.4456 \mathrm{E}\end{array}$ \\
\hline & $\underline{\text { CCDB38233 A08 }}$ & DCCDB008-21 & & $\begin{array}{l}\text { Kolašin, Kolašinska } \\
\text { rijeka stream }\end{array}$ & $\begin{array}{l}42.8391 \mathrm{~N} \\
19.5749 \mathrm{E}\end{array}$ \\
\hline & CCDB38233 A09 & DCCDB009-21 & & $\begin{array}{l}\text { Kolašin, Kolašinska } \\
\text { rijeka stream }\end{array}$ & $\begin{array}{l}42.8391 \mathrm{~N} \\
19.5749 \mathrm{E}\end{array}$ \\
\hline & $\underline{\text { CCDB38233 A10 }}$ & DCCDB010-21 & & $\begin{array}{l}\text { Kolaši, Kolašinska } \\
\text { rijeka stream }\end{array}$ & $\begin{array}{l}42.8391 \mathrm{~N} \\
19.5749 \mathrm{E}\end{array}$ \\
\hline \multirow[t]{3}{*}{$\begin{array}{l}\text { Protzia squamosa } \\
\text { paucipora }\end{array}$} & $\underline{\text { CCDB } 38361 \mathrm{~A} 09}$ & DCDDJ009-21 & \multirow[t]{3}{*}{ BOLD:AEL1015 } & $\begin{array}{l}\text { Kolašin, spring on } \\
\text { road to Trešnjevik }\end{array}$ & $\begin{array}{l}42.7405 \mathrm{~N} \\
19.6801 \mathrm{E}\end{array}$ \\
\hline & $\underline{\text { CCDB } 38361 \mathrm{~A} 10}$ & DCDDJ010-21 & & $\begin{array}{l}\text { Kolašin, spring on } \\
\text { road to Trešnjevik }\end{array}$ & $\begin{array}{l}42.7405 \mathrm{~N} \\
19.6801 \mathrm{E}\end{array}$ \\
\hline & CCDB $38361 \mathrm{~A} 11$ & DCDDJ011-21 & & $\begin{array}{l}\text { Kolašin, spring on } \\
\text { road to Trešnjevik }\end{array}$ & $\begin{array}{l}42.7405 \mathrm{~N} \\
19.6801 \mathrm{E}\end{array}$ \\
\hline Protzia octopora & $\underline{\text { CCDB38233 D09 }}$ & DCCDB045-21 & BOLD:AEI5747 & $\begin{array}{l}\text { Kolašin, Bistrica } \\
\text { stream }\end{array}$ & $\begin{array}{l}42.9871 \mathrm{~N} \\
19.4338 \mathrm{E}\end{array}$ \\
\hline \multirow[t]{4}{*}{ Protzia halberti } & Hyd MN VP7 & DNAEC081-20 & \multirow[t]{4}{*}{ BOLD:AED9646 } & $\begin{array}{l}\text { Bijelo Polje, } \\
\text { Lještanica stream }\end{array}$ & $\begin{array}{l}43.0631 \mathrm{~N} \\
19.5808 \mathrm{E}\end{array}$ \\
\hline & 3. CG2020 82 & DNAEC002-20 & & $\begin{array}{l}\text { Bijelo Polje, } \\
\text { Lještanica stream }\end{array}$ & $\begin{array}{l}43.0631 \mathrm{~N} \\
19.5808 \mathrm{E}\end{array}$ \\
\hline & 4. CG2020 1 & DNAEC003-20 & & $\begin{array}{l}\text { Bijelo Polje, } \\
\text { Lještanica stream }\end{array}$ & $\begin{array}{l}43.0631 \mathrm{~N} \\
19.5808 \mathrm{E}\end{array}$ \\
\hline & 5. CG2020 13 & DNAEC004-20 & & $\begin{array}{l}\text { Bijelo Polje, } \\
\text { Lještanica stream }\end{array}$ & $\begin{array}{l}43.0631 \mathrm{~N} \\
19.5808 \mathrm{E}\end{array}$ \\
\hline
\end{tabular}




\begin{tabular}{|c|c|c|c|c|c|}
\hline Taxa & Voucher Code & $\begin{array}{l}\text { BOLD } \\
\text { Process } \\
\text { ID }\end{array}$ & BIN & Locality & Coordinates \\
\hline \multirow[t]{3}{*}{ Protzia rotunda } & 6. M18 $01 \_1 \quad D 10$ & DNAEC045-20 & \multirow[t]{3}{*}{ BOLD:AED8976 } & $\begin{array}{l}\text { Žabljak, Sedlo, } \\
\text { spring Studenac }\end{array}$ & $\begin{array}{l}43.0973 \mathrm{~N} \\
19.0702 \mathrm{E}\end{array}$ \\
\hline & CCDB-3867-E04 & DNCBD052-20 & & $\begin{array}{l}\text { Bar, Međurječka } \\
\text { rijeka stream }\end{array}$ & $\begin{array}{l}42.0363 \mathrm{~N}, \\
19.2179 \mathrm{E}\end{array}$ \\
\hline & CCDB-3867-E05 & DNCBD053-20 & & $\begin{array}{l}\text { Bar, Međurječka } \\
\text { rijeka stream }\end{array}$ & $\begin{array}{l}42.0363 \mathrm{~N} \\
19.2179 \mathrm{E}\end{array}$ \\
\hline \multirow[t]{3}{*}{ Protzia rugosa } & 6. CG2020_1_4 & DNAEC005-20 & \multirow[t]{3}{*}{ BOLD:AEE010 } & $\begin{array}{l}\text { Bijelo Polje, } \\
\text { Lještanica stream }\end{array}$ & $\begin{array}{l}43.0631 \mathrm{~N} \\
19.5808 \mathrm{E}\end{array}$ \\
\hline & 7. CG2020 8 B6 & DNAEC017-20 & & $\begin{array}{l}\text { Berane, spring nr } \\
\text { Mon. Djurdjevi } \\
\text { Stupovi }\end{array}$ & $\begin{array}{l}42.8527 \mathrm{~N} \\
19.862 \mathrm{E}\end{array}$ \\
\hline & $\underline{\text { CCDB38233 D05 }}$ & DCCDB041-21 & & $\begin{array}{l}\text { Mojkovac, Bistrica } \\
\text { stream }\end{array}$ & $\begin{array}{l}42.9871 \mathrm{~N} \\
19.4338 \mathrm{E}\end{array}$ \\
\hline \multicolumn{6}{|l|}{ Hydrodromidae } \\
\hline $\begin{array}{l}\text { Hydrodroma } \\
\text { reinhardi }\end{array}$ & CCDB-3867-G04 & DNCBD076-20 & BOLD:AEF0798 & $\begin{array}{l}\text { Podgorica, Cijevna } \\
\text { River at Dinoša }\end{array}$ & $\begin{array}{l}42.4057 \mathrm{~N} \\
19.3569 \mathrm{E}\end{array}$ \\
\hline $\begin{array}{l}\text { Hydrodroma } \\
\text { torrenticola }\end{array}$ & CCDB-3867-E06 & DNCBD054-20 & BOLD:AEF3799 & $\begin{array}{l}\text { Bar, Medjurječka } \\
\text { rijeka stream }\end{array}$ & $\begin{array}{l}42.0363 \mathrm{~N} \\
19.2179 \mathrm{E}\end{array}$ \\
\hline \multicolumn{6}{|l|}{ Lebertiidae } \\
\hline \multirow[t]{4}{*}{ Lebertia jadrensis } & CCDB 38361 C09 & DCDDJ033-21 & \multirow[t]{4}{*}{ BOLD:ADK0383 } & $\begin{array}{l}\text { Kolašin, Bistrica } \\
\text { stream at Crkvine }\end{array}$ & $\begin{array}{l}42.8054 \mathrm{~N} \\
19.4456 \mathrm{E}\end{array}$ \\
\hline & CCDB-3867-G08 & DNCBD080-20 & & $\begin{array}{l}\text { Podgorica, Cijevna } \\
\text { River at Dinoša }\end{array}$ & $\begin{array}{l}42.4057 \mathrm{~N}, \\
19.3569 \mathrm{E}\end{array}$ \\
\hline & $\underline{\mathrm{CCDB}} 38361 \mathrm{C08}$ & DCDDJ032-21 & & $\begin{array}{l}\text { Kolašin, Bistrica } \\
\text { stream at Crkvine }\end{array}$ & $\begin{array}{l}42.8054 \mathrm{~N} \\
19.4456 \mathrm{E}\end{array}$ \\
\hline & CCDB-3867-F10 & DNCBD070-20 & & $\begin{array}{l}\text { Danilovgrad, spring } \\
\text { below the bridge }\end{array}$ & $\begin{array}{l}42.5542 \mathrm{~N} \\
19.1059 \mathrm{E}\end{array}$ \\
\hline Lebertia cuneifera & CCDB 38363 A01 & $\underline{\text { SEPTA001-21 }}$ & BOLD:ADV4392 & $\begin{array}{l}\text { Nikšić, spring } \\
\text { "Babino sicelo" }\end{array}$ & $\begin{array}{l}42.8043 \mathrm{~N} \\
19.2152 \mathrm{E}\end{array}$ \\
\hline \multirow[t]{6}{*}{ Lebertia variolata } & CCDB-3867-B05 & DNCBD017-20 & \multirow[t]{6}{*}{ BOLD:ADK0996 } & $\begin{array}{l}\text { Bar, stream in } \\
\text { Godinje Village }\end{array}$ & $\begin{array}{l}42.2206 \mathrm{~N} \\
19.1118 \mathrm{E}\end{array}$ \\
\hline & CCDB-3867-B07 & DNCBD019-20 & & $\begin{array}{l}\text { Bar, stream in } \\
\text { Godinje Village }\end{array}$ & $\begin{array}{l}42.2206 \mathrm{~N} \\
19.1118 \mathrm{E}\end{array}$ \\
\hline & $\underline{\text { CCDB-3867-D03 }}$ & DNCBD039-20 & & $\begin{array}{l}\text { Bar, Rikavac stream } \\
\text { above Old Bar }\end{array}$ & $\begin{array}{l}42.1001 \mathrm{~N} \\
19.1432 \mathrm{E}\end{array}$ \\
\hline & CCDB-3867-D04 & DNCBD040-20 & & $\begin{array}{l}\text { Bar, Rikavac stream } \\
\text { above Old Bar }\end{array}$ & $\begin{array}{l}42.1001 \mathrm{~N} \\
19.1432 \mathrm{E}\end{array}$ \\
\hline & CCDB-3867-D05 & DNCBD041-20 & & $\begin{array}{l}\text { Bar, Rikavac stream } \\
\text { above Old Bar }\end{array}$ & $\begin{array}{l}42.1001 \mathrm{~N} \\
19.1432 \mathrm{E}\end{array}$ \\
\hline & CCDB-3867-D06 & DNCBD042-20 & & $\begin{array}{l}\text { Bar, Rikavac stream } \\
\text { above Old Bar }\end{array}$ & $\begin{array}{l}\text { 42.1001N, } \\
19.1432 \mathrm{E}\end{array}$ \\
\hline
\end{tabular}




\begin{tabular}{|c|c|c|c|c|c|}
\hline Taxa & Voucher Code & $\begin{array}{l}\text { BOLD } \\
\text { Process } \\
\text { ID }\end{array}$ & BIN & Locality & Coordinates \\
\hline & 16. M19 $24 \quad 3 \quad E 7$ & DNAEC054-20 & & $\begin{array}{l}\text { Bar, Medjurječka } \\
\text { rijeka stream }\end{array}$ & $\begin{array}{l}42.0226 \mathrm{~N} \\
19.22 \mathrm{E}\end{array}$ \\
\hline & 17. M19 $24 \quad 3$ E8 & DNAEC055-20 & & $\begin{array}{l}\text { Bar, Medjurječka } \\
\text { rijeka stream }\end{array}$ & $\begin{array}{l}42.0226 \mathrm{~N} \\
19.22 \mathrm{E}\end{array}$ \\
\hline \multirow[t]{3}{*}{ Lebertia natans } & $\underline{\mathrm{CCDB} 38233 \mathrm{~F} 03}$ & $\underline{\text { DCCDB063-21 }}$ & \multirow[t]{3}{*}{ BOLD:AEF5684 } & $\begin{array}{l}\text { Danilovgrad, spring } \\
\text { below the bridge }\end{array}$ & $\begin{array}{l}42.5541 \mathrm{~N} \\
19.1057 \mathrm{E}\end{array}$ \\
\hline & CCDB38233 F04 & DCCDB064-21 & & $\begin{array}{l}\text { Danilovgrad, spring } \\
\text { below the bridge }\end{array}$ & $\begin{array}{l}42.5541 \mathrm{~N} \\
19.1057 \mathrm{E}\end{array}$ \\
\hline & CCDB-3867-F06 & DNCBD066-20 & & $\begin{array}{l}\text { Danilovgrad, spring } \\
\text { below the bridge }\end{array}$ & $\begin{array}{l}42.5542 \mathrm{~N} \\
19.1059 \mathrm{E}\end{array}$ \\
\hline \multirow[t]{6}{*}{ Lebertia glabra } & $\underline{\text { CCDB38233 C04 }}$ & $\underline{\mathrm{DCCDB} 028-21}$ & \multirow[t]{3}{*}{ BOLD:AEI2925 } & $\begin{array}{l}\text { Kolašin, Kolašinska } \\
\text { rijeka stream }\end{array}$ & $\begin{array}{l}42.8391 \mathrm{~N} \\
19.5749 \mathrm{E}\end{array}$ \\
\hline & $\underline{\text { CCDB38233 D03 }}$ & $\underline{\text { DCCDB039-21 }}$ & & $\begin{array}{l}\text { Kolašin, Bistrica } \\
\text { stream at Crkvine }\end{array}$ & $\begin{array}{l}42.9871 \mathrm{~N} \\
19.4338 \mathrm{E}\end{array}$ \\
\hline & $\underline{\text { CCDB38233 D04 }}$ & DCCDB040-21 & & $\begin{array}{l}\text { Kolašin, Bistrica } \\
\text { stream at Crkvine }\end{array}$ & $\begin{array}{l}42.9871 \mathrm{~N} \\
19.4338 \mathrm{E}\end{array}$ \\
\hline & $\underline{\text { CCDB38233 D01 }}$ & $\underline{\text { DCCDB037-21 }}$ & \multirow[t]{2}{*}{ BOLD:ACS0595 } & $\begin{array}{l}\text { Mojkovac, Bistrica } \\
\text { stream }\end{array}$ & $\begin{array}{l}42.9871 \mathrm{~N} \\
19.4338 \mathrm{E}\end{array}$ \\
\hline & CCDB38233 D02 & DCCDB038-21 & & $\begin{array}{l}\text { Mojkovac, Bistrica } \\
\text { stream }\end{array}$ & $\begin{array}{l}42.9871 \mathrm{~N} \\
19.4338 \mathrm{E}\end{array}$ \\
\hline & $\underline{\mathrm{CCDB} 38233 \mathrm{C05}}$ & DCCDB029-21 & BOLD:ACR9598 & $\begin{array}{l}\text { Kolašin, Kolašinska } \\
\text { rijeka stream }\end{array}$ & $\begin{array}{l}42.8391 \mathrm{~N} \\
19.5749 \mathrm{E}\end{array}$ \\
\hline \multirow[t]{8}{*}{ Lebertia inaequalis } & $\underline{\text { CCDB-3867-C03 }}$ & DNCBD027-20 & BOLD:AEF5913 & Tuzi, Vitoja, pool & $\begin{array}{l}42.324 \mathrm{~N} \\
19.3637 \mathrm{E}\end{array}$ \\
\hline & CCDB-3867-B11 & DNCBD023-20 & \multirow[t]{6}{*}{ BOLD:ADF6223 } & Tuzi, Vitoja, pool & $\begin{array}{l}42.324 \mathrm{~N} \\
19.3637 \mathrm{E}\end{array}$ \\
\hline & CCDB-3867-C02 & DNCBD026-20 & & Tuzi, Vitoja, pool & $\begin{array}{l}42.324 \mathrm{~N} \\
19.3637 \mathrm{E}\end{array}$ \\
\hline & $\underline{\text { CCDB } 38363 \text { B04 }}$ & SEPTA016-21 & & $\begin{array}{l}\text { Bar, Skadar Lake at } \\
\text { Murići }\end{array}$ & $\begin{array}{l}42.1637 \mathrm{~N} \\
19.2214 \mathrm{E}\end{array}$ \\
\hline & $\underline{\text { CCDB } 38363 \text { B06 }}$ & SEPTA018-21 & & $\begin{array}{l}\text { Bar, Skadar Lake at } \\
\text { Murići }\end{array}$ & $\begin{array}{l}42.1637 \mathrm{~N} \\
19.2214 \mathrm{E}\end{array}$ \\
\hline & CCDB 38363 B10 & SEPTA022-21 & & $\begin{array}{l}\text { Podgorica, Skadar } \\
\text { Lake at Donja } \\
\text { Plavnica }\end{array}$ & $\begin{array}{l}42.2724 \mathrm{~N} \\
19.2007 \mathrm{E}\end{array}$ \\
\hline & $\underline{\text { CCDB } 38363 \text { B11 }}$ & $\underline{\text { SEPTA023-21 }}$ & & $\begin{array}{l}\text { Podgorica, Gornja } \\
\text { Plavnica, river }\end{array}$ & $\begin{array}{l}42.2889 \mathrm{~N} \\
19.2108 \mathrm{E}\end{array}$ \\
\hline & $\underline{\text { CCDB-3867-E12 }}$ & DNCBD060-20 & BOLD:AEF2742 & $\begin{array}{l}\text { Bar, Medjurjecka } \\
\text { rijeka stream }\end{array}$ & $\begin{array}{l}42.0363 \mathrm{~N} \\
19.2179 \mathrm{E}\end{array}$ \\
\hline Lebertia insignis & $\underline{\text { CCDB38233 B12 }}$ & DCCDB024-21 & BOLD:AEB9107 & $\begin{array}{l}\text { Danilovgrad, River } \\
\text { Zeta near Slap }\end{array}$ & $\begin{array}{l}42.6001 \mathrm{~N} \\
19.0656 \mathrm{E}\end{array}$ \\
\hline Lebertia maculosa & $\begin{array}{l}\underline{32 .} \\
\underline{\mathrm{CG}} 2020 \quad 1 \quad \mathrm{C} 11 \\
\end{array}$ & DNAEC033-20 & BOLD:AED9197 & $\begin{array}{l}\text { Bijelo Polje, } \\
\text { Lještanica stream }\end{array}$ & $\begin{array}{l}43.0631 \mathrm{~N} \\
19.5809 \mathrm{E}\end{array}$ \\
\hline
\end{tabular}




\begin{tabular}{|c|c|c|c|c|c|}
\hline Taxa & Voucher Code & $\begin{array}{l}\text { BOLD } \\
\text { Process } \\
\text { ID }\end{array}$ & BIN & Locality & Coordinates \\
\hline & $\begin{array}{l}33 . \\
\mathrm{CG} 2020 \quad 1 \quad \mathrm{C} 12\end{array}$ & DNAEC034-2 & & $\begin{array}{l}\text { Bijelo Polje, } \\
\text { Lještanica stream }\end{array}$ & $\begin{array}{l}43.0631 \mathrm{~N} \\
19.5809 \mathrm{E}\end{array}$ \\
\hline & 1. CG2020 8 & DNAEC001-20 & BOLD:AED9718 & $\begin{array}{l}\text { Berane, spring nr. } \\
\text { Mon. Djurdjevi } \\
\text { Stupovi }\end{array}$ & $\begin{array}{l}42.8527 \mathrm{~N} \\
19.862 \mathrm{E}\end{array}$ \\
\hline & $\underline{\mathrm{CCDB} 38361 \mathrm{H} 01}$ & $\underline{\text { DCDDJ085-21 }}$ & & $\begin{array}{l}\text { Kolašin, spring at } \\
\text { Monastir Morača }\end{array}$ & $\begin{array}{l}42.7668 \mathrm{~N} \\
19.3906 \mathrm{E}\end{array}$ \\
\hline \multirow[t]{4}{*}{ Lebertia porosa } & $\underline{\text { CCDB-3867-G09 }}$ & DNCBD081-20 & \multirow[t]{2}{*}{ BOLD:ACS0974 } & $\begin{array}{l}\text { Podgorica, Cijevna } \\
\text { River at Dinoša }\end{array}$ & $\begin{array}{l}42.4057 \mathrm{~N} \\
19.3569 \mathrm{E}\end{array}$ \\
\hline & $\underline{\text { CCDB } 38363 \text { C10 }}$ & $\underline{\text { SEPTA034-21 }}$ & & $\begin{array}{l}\text { Cetinje, River } \\
\text { Crnojevića }\end{array}$ & $\begin{array}{l}42.3557 \mathrm{~N} \\
19.0228 \mathrm{E}\end{array}$ \\
\hline & $\underline{\text { CCDB38233 A01 }}$ & $\underline{\text { DCCDB001-21 }}$ & \multirow[t]{2}{*}{ BOLD:AED4662 } & $\begin{array}{l}\text { Podgorica, spring } \\
\text { Mareza }\end{array}$ & $\begin{array}{l}42.4801 \mathrm{~N} \\
19.1822 \mathrm{E}\end{array}$ \\
\hline & 7. CG2020 10 & DNAEC006-20 & & Tuzi, Vitoja spring & $\begin{array}{l}42.3254 \mathrm{~N} \\
19.3628 \mathrm{E}\end{array}$ \\
\hline \multirow[t]{3}{*}{ Lebertia reticulata } & Hyd MN VP13 & DNAEC086-20 & \multirow[t]{3}{*}{ BOLD:ADT9218 } & $\begin{array}{l}\text { Šavnik, spring of } \\
\text { Bukovica stream }\end{array}$ & $\begin{array}{l}43.0589 \mathrm{~N} \\
19.1103 \mathrm{E}\end{array}$ \\
\hline & $\underline{\text { Hyd MN VP14 }}$ & DNAEC087-20 & & $\begin{array}{l}\text { Šavnik, spring of } \\
\text { Bukovica stream }\end{array}$ & $\begin{array}{l}43.0589 \mathrm{~N} \\
19.1103 \mathrm{E}\end{array}$ \\
\hline & CCDB 38363 A11 & SEPTA011-21 & & $\begin{array}{l}\text { Nikšić, spring } \\
\text { Vukovo Vrelo }\end{array}$ & $\begin{array}{l}42.8574 \mathrm{~N} \\
18.9426 \mathrm{E}\end{array}$ \\
\hline \multirow[t]{2}{*}{ Lebertia schechteli } & 9. CG2020 & DNAEC008-20 & \multirow[t]{2}{*}{ BOLD:AED9612 } & $\begin{array}{l}\text { Žabljak, Sedlo, } \\
\text { spring Studenac }\end{array}$ & $\begin{array}{l}43.0973 \mathrm{~N} \\
19.0702 \mathrm{E}\end{array}$ \\
\hline & 10. CG2020 23 & DNAEC009-20 & & $\begin{array}{l}\text { Žabljak, Sedlo, } \\
\text { spring Studenac }\end{array}$ & $\begin{array}{l}43.0973 \mathrm{~N} \\
19.0702 \mathrm{E}\end{array}$ \\
\hline \multicolumn{6}{|l|}{ Oxidae } \\
\hline \multirow[t]{4}{*}{ Oxus angustipositus } & $\underline{\text { CCDB } 38361 \mathrm{C} 03}$ & DCDDJ027-21 & \multirow[t]{4}{*}{ BOLD:AEB9099 } & Ulcinj, Šasko Lake & $\begin{array}{l}41.9768 \mathrm{~N} \\
19.3388 \mathrm{E}\end{array}$ \\
\hline & $\underline{\text { CCDB-38679-A11 }}$ & $\underline{\text { DNCBD011-20 }}$ & & $\begin{array}{l}\text { Cetinje, Poseljanski } \\
\text { stream, lower part }\end{array}$ & $\begin{array}{l}42.3057 \mathrm{~N} \\
19.0557 \mathrm{E}\end{array}$ \\
\hline & $\underline{\text { CCDB } 38363 \text { B05 }}$ & SEPTA017-21 & & $\begin{array}{l}\text { Bar, Skadar Lake at } \\
\text { Murići }\end{array}$ & $\begin{array}{l}42.1637 \mathrm{~N} \\
19.2214 \mathrm{E}\end{array}$ \\
\hline & $\underline{\text { CCDB } 38363 \text { B07 }}$ & SEPTA019-21 & & $\begin{array}{l}\text { Bar, Skadar Lake at } \\
\text { Murići }\end{array}$ & $\begin{array}{l}42.1637 \mathrm{~N} \\
19.2214 \mathrm{E}\end{array}$ \\
\hline \multicolumn{6}{|l|}{ Teutoniidae } \\
\hline \multirow[t]{2}{*}{ Teutonia cometes } & $\begin{array}{l}\frac{33 .}{M 19} \quad 20 \quad 3 \quad F 11 \\
\end{array}$ & DNAEC068-20 & \multirow[t]{2}{*}{ BOLD:ACH7884 } & $\begin{array}{l}\text { Podgorica, Mareza } \\
\text { canal }\end{array}$ & $\begin{array}{l}42.479 \mathrm{~N} \\
19.1813 \mathrm{E}\end{array}$ \\
\hline & Hyd MN VP5 & DNAEC079-20 & & $\begin{array}{l}\text { Danilovgrad, spring } \\
\text { Svinjiška vrela }\end{array}$ & $\begin{array}{l}42.6384 \mathrm{~N} \\
19.0074 \mathrm{E}\end{array}$ \\
\hline \multicolumn{6}{|l|}{ Sperchontidae } \\
\hline $\begin{array}{l}\text { Sperchon } \\
\text { brevirostris }\end{array}$ & $\underline{\mathrm{CCDB} 38233 \mathrm{D} 07}$ & DCCDB043-21 & BOLD:ACP6107 & $\begin{array}{l}\text { Mojkovac, Bistrica } \\
\text { stream }\end{array}$ & $\begin{array}{l}42.9871 \mathrm{~N} \\
19.4338 \mathrm{E}\end{array}$ \\
\hline
\end{tabular}




\begin{tabular}{|c|c|c|c|c|c|}
\hline Taxa & Voucher Code & $\begin{array}{l}\text { BOLD } \\
\text { Process } \\
\text { ID }\end{array}$ & BIN & Locality & Coordinates \\
\hline & CCDB38233 D08 & DCCDB044-21 & BOLD:AED3857 & $\begin{array}{l}\text { Mojkovac, Bistrica } \\
\text { stream }\end{array}$ & $\begin{array}{l}42.9871 \mathrm{~N} \\
19.4338 \mathrm{E}\end{array}$ \\
\hline & $\underline{\text { CCDB38233 A11 }}$ & DCCDB011-21 & & $\begin{array}{l}\text { Kolašin, Kolašinska } \\
\text { rijeka stream }\end{array}$ & $\begin{array}{l}42.8391 \mathrm{~N} \\
19.5749 \mathrm{E}\end{array}$ \\
\hline \multirow[t]{2}{*}{ Sperchon clupeifer } & Hyd MN VP11 & DNAEC084-20 & BOLD:AEE4061 & $\begin{array}{l}\text { Žabljak, Ljutica } \\
\text { stream }\end{array}$ & $\begin{array}{l}43.1378 \mathrm{~N} \\
19.3023 \mathrm{E}\end{array}$ \\
\hline & CCDB-3867-B04 & DNCBD016-20 & BOLD:ACS1100 & $\begin{array}{l}\text { Bar, stream in } \\
\text { Godinje Village }\end{array}$ & $\begin{array}{l}42.2206 \mathrm{~N} \\
19.1118 \mathrm{E}\end{array}$ \\
\hline Sperchon hibernicus & CCDB-3867-D02 & DNCBD038-20 & BOLD:AEF3824 & $\begin{array}{l}\text { Bar, Rikavac stream } \\
\text { above Old Bar }\end{array}$ & $\begin{array}{l}42.1001 \mathrm{~N} \\
19.1432 \mathrm{E}\end{array}$ \\
\hline \multirow[t]{2}{*}{ Sperchon hispidus } & 12. M19 29A 8 E3 & DNAEC050-20 & \multirow[t]{2}{*}{ BOLD:AED3202 } & $\begin{array}{l}\text { Danilovgrad, Zeta } \\
\text { River at Spuž }\end{array}$ & $\begin{array}{l}42.5113 \mathrm{~N} \\
19.1982 \mathrm{E}\end{array}$ \\
\hline & $\begin{array}{l}\frac{29 .}{\mathrm{CG}} 20207 \mathrm{C} 8 \mathrm{C} 7 \\
.\end{array}$ & DNAEC030-20 & & $\begin{array}{l}\text { Danilovgrad, Zeta } \\
\text { River at Spuž }\end{array}$ & $\begin{array}{l}42.5113 \mathrm{~N} \\
19.1982 \mathrm{E}\end{array}$ \\
\hline $\begin{array}{l}\text { Spechon } \\
\text { denticulatus }\end{array}$ & 10. CG2020 8 B8 & DNAEC019-20 & BOLD:AED8428 & $\begin{array}{l}\text { Berane, spring nr. } \\
\text { Mon. Djurdjevi } \\
\text { Stupovi }\end{array}$ & $\begin{array}{l}42.8527 \mathrm{~N} \\
19.862 \mathrm{E}\end{array}$ \\
\hline Sperchon papillosus & 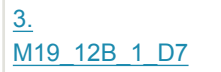 & DNAEC043-20 & BOLD:AED2134 & $\begin{array}{l}\text { Budva, Lastva } \\
\text { Grbaljska, stream }\end{array}$ & $\begin{array}{l}42.3103 \mathrm{~N} \\
18.8138 \mathrm{E}\end{array}$ \\
\hline \multirow[t]{6}{*}{$\begin{array}{l}\text { Sperchon } \\
\text { thienemanni }\end{array}$} & Hyd MN VP4 & DNAEC078-20 & \multirow[t]{6}{*}{ BOLD:ADV4077 } & $\begin{array}{l}\text { Šavnik, spring } \\
\text { Kikov izvor near } \\
\text { Boan }\end{array}$ & $\begin{array}{l}42.9465 \mathrm{~N} \\
19.1893 \mathrm{E}\end{array}$ \\
\hline & Hyd MN VP10 & DNAEC083-20 & & $\begin{array}{l}\text { Žabljak, Sedlo, } \\
\text { Studenac spring }\end{array}$ & $\begin{array}{l}43.0972 \mathrm{~N} \\
19.0702 \mathrm{E}\end{array}$ \\
\hline & $\underline{\mathrm{CCDB}} 38361 \mathrm{~A} 03$ & DCDDJ003-21 & & $\begin{array}{l}\text { Kolašin, Lalevića } \\
\text { Dolovi, spring \#1 }\end{array}$ & $\begin{array}{l}42.899 \mathrm{~N} \\
19.631 \mathrm{E}\end{array}$ \\
\hline & CCDB $38363 \mathrm{~A} 02$ & SEPTA002-21 & & $\begin{array}{l}\text { Nikšić, Lukavica } \\
\text { Mt., spring Babino } \\
\text { Sicelo }\end{array}$ & $\begin{array}{l}42.8043 \mathrm{~N} \\
19.2152 \mathrm{E}\end{array}$ \\
\hline & CCDB $38363 \mathrm{~A} 04$ & SEPTA004-21 & & $\begin{array}{l}\text { Nikšić, Lukavica } \\
\text { Mt., spring Babino } \\
\text { Sicelo }\end{array}$ & $\begin{array}{l}42.8043 \mathrm{~N} \\
19.2152 \mathrm{E}\end{array}$ \\
\hline & $\underline{\text { CCDB } 38363 \text { A05 }}$ & SEPTA005-21 & & $\begin{array}{l}\text { Nikšić, Lukavica } \\
\text { Mt., spring Babino } \\
\text { Sicelo }\end{array}$ & $\begin{array}{l}42.8043 \mathrm{~N} \\
19.2152 \mathrm{E}\end{array}$ \\
\hline \multirow[t]{4}{*}{ Sperchon violaceus } & $\underline{\text { Hyd MN VP8 }}$ & DNAEC088-20 & \multirow[t]{4}{*}{ BOLD:AAN0076 } & $\begin{array}{l}\text { Žabljak, Mlinski } \\
\text { potok stream }\end{array}$ & $\begin{array}{l}43.1494 \mathrm{~N} \\
19.0898 \mathrm{E}\end{array}$ \\
\hline & $\begin{array}{l}\frac{27 .}{\mathrm{M} 19} \quad 16 \mathrm{~A} \\
\end{array}$ & DNAEC062- & & $\begin{array}{l}\text { Kolašin, Biogradska } \\
\text { River }\end{array}$ & $\begin{array}{l}42.8968 \mathrm{~N} \\
19.6047 \mathrm{E}\end{array}$ \\
\hline & 56. CG20201 & DNAEC010-20 & & $\begin{array}{l}\text { Bijelo Polje, } \\
\text { Lještanica stream }\end{array}$ & $\begin{array}{l}43.0631 \mathrm{~N} \\
19.5809 \mathrm{E}\end{array}$ \\
\hline & 57. CG2020 8 & DNAEC011-20 & & $\begin{array}{l}\text { Bijelo Polje, } \\
\text { Lještanica stream }\end{array}$ & $\begin{array}{l}43.0631 \mathrm{~N} \\
19.5809 \mathrm{E}\end{array}$ \\
\hline
\end{tabular}




\begin{tabular}{|c|c|c|c|c|c|}
\hline \multirow[t]{7}{*}{ Taxa } & Voucher Code & $\begin{array}{l}\text { BOLD } \\
\text { Process } \\
\text { ID }\end{array}$ & BIN & Locality & Coordinates \\
\hline & 58. CG2020 & DNAEC012-20 & & $\begin{array}{l}\text { Bijelo Polje, } \\
\text { Lještanica stream }\end{array}$ & $\begin{array}{l}43.0631 \mathrm{~N} \\
19.5809 \mathrm{E}\end{array}$ \\
\hline & 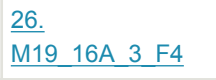 & DNAEC061-20 & & $\begin{array}{l}\text { Kolašin, Biogradska } \\
\text { River }\end{array}$ & $\begin{array}{l}42.8968 \mathrm{~N} \\
19.6047 \mathrm{E}\end{array}$ \\
\hline & $\begin{array}{l}\frac{28 .}{M 19} \quad 16 A \quad 3 \quad F 6 \\
\end{array}$ & DNAEC063-20 & & $\begin{array}{l}\text { Kolašin, Biogradska } \\
\text { River }\end{array}$ & $\begin{array}{l}42.8968 \mathrm{~N} \\
19.6047 \mathrm{E}\end{array}$ \\
\hline & $\underline{\text { CCDB38233 D06 }}$ & DCCDB042-21 & & $\begin{array}{l}\text { Mojkovac, Bistrica } \\
\text { stream }\end{array}$ & $\begin{array}{l}42.9871 \mathrm{~N} \\
19.4338 \mathrm{E}\end{array}$ \\
\hline & $\underline{\text { CCDB38233 H10 }}$ & DCCDB094-21 & & $\begin{array}{l}\text { Mojkovac, spring in } \\
\text { Bistrica Village }\end{array}$ & $\begin{array}{l}42.9862 \mathrm{~N} \\
19.4349 \mathrm{E}\end{array}$ \\
\hline & $\underline{\text { CCDB38233 H11 }}$ & $\underline{\text { DCCDB095-21 }}$ & & $\begin{array}{l}\text { Mojkovac, spring in } \\
\text { Bistrica Village }\end{array}$ & $\begin{array}{l}42.9862 \mathrm{~N} \\
19.4349 \mathrm{E}\end{array}$ \\
\hline \multirow[t]{2}{*}{$\begin{array}{l}\text { Sperchonopsis } \\
\text { verrucosa }\end{array}$} & $\underline{\text { CCDB } 38361 \text { B11 }}$ & DCDDJ023-21 & BOLD:AEK8297 & $\begin{array}{l}\text { Cetinje, spring } \\
\text { "Smokov Vijenac" }\end{array}$ & $\begin{array}{l}42.254 \mathrm{~N} \\
18.9902 \mathrm{E}\end{array}$ \\
\hline & 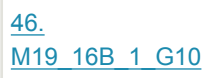 & DNAEC040-20 & BOLD:ACS9705 & $\begin{array}{l}\text { Kolašin, Biogradska } \\
\text { River }\end{array}$ & $\begin{array}{l}42.8968 \mathrm{~N} \\
19.6047 \mathrm{E}\end{array}$ \\
\hline \multicolumn{6}{|l|}{ Torrenticolidae } \\
\hline \multirow[t]{3}{*}{$\begin{array}{l}\text { Monatractides } \\
\text { madritensis }\end{array}$} & $\underline{\text { CCDB-3867-G11 }}$ & DNCBD083-20 & BOLD:AED3803 & $\begin{array}{l}\text { Podgorica, Cijevna } \\
\text { River at Dinoša }\end{array}$ & $\begin{array}{l}42.4057 \mathrm{~N} \\
19.3569 \mathrm{E}\end{array}$ \\
\hline & $\begin{array}{l}\frac{44 .}{\mathrm{M} 19} \quad 12 \mathrm{~B} \quad 3 \quad \mathrm{G} 8 \\
\end{array}$ & DNAEC $075-20$ & & $\begin{array}{l}\text { Budva, Lastva } \\
\text { Grbaljska, first } \\
\text { order stream }\end{array}$ & $\begin{array}{l}42.3103 \mathrm{~N} \\
18.8138 \mathrm{E}\end{array}$ \\
\hline & $\underline{\text { CCDB-3867-B01 }}$ & $\underline{\text { DNCBD013-20 }}$ & BOLD:AEL3852 & $\begin{array}{l}\text { Bar, stream in } \\
\text { Godinje Village }\end{array}$ & $\begin{array}{l}42.2206 \mathrm{~N} \\
19.1118 \mathrm{E}\end{array}$ \\
\hline \multirow[t]{2}{*}{$\begin{array}{l}\text { Monatractides } \\
\text { stadleri }\end{array}$} & $\underline{\mathrm{CCDB} 38233 \mathrm{CO} 3}$ & $\underline{\text { DCCDB027-21 }}$ & BOLD:AED3802 & $\begin{array}{l}\text { Bar, Rikavac stream } \\
\text { above Old Bar }\end{array}$ & $\begin{array}{l}42.1001 \mathrm{~N} \\
19.1432 \mathrm{E}\end{array}$ \\
\hline & $\begin{array}{llll}\frac{45 .}{\mathrm{M} 19} & 129 & 3 & \mathrm{G} 9 \\
\end{array}$ & DNAEC076-20 & & $\begin{array}{l}\text { Budva, Lastva } \\
\text { Grbaljska, first } \\
\text { order stream }\end{array}$ & $\begin{array}{l}42.3103 \mathrm{~N} \\
18.8138 \mathrm{E}\end{array}$ \\
\hline \multirow[t]{3}{*}{ Torrenticola amplexa } & $\underline{\text { CCDB-3867-F08 }}$ & DNCBD068-20 & BOLD:ACR0665 & $\begin{array}{l}\text { Danilovgrad, spring } \\
\text { below the bridge }\end{array}$ & $\begin{array}{l}42.5542 \mathrm{~N} \\
19.1059 \mathrm{E}\end{array}$ \\
\hline & CCDB-3867-F09 & DNCBD069-20 & & $\begin{array}{l}\text { Danilovgrad, spring } \\
\text { below the bridge }\end{array}$ & $\begin{array}{l}42.5542 \mathrm{~N} \\
19.1059 \mathrm{E}\end{array}$ \\
\hline & $\underline{\text { CCDB38233 G04 }}$ & DCCDB076-21 & & $\begin{array}{l}\text { Danilovgrad, spring } \\
\text { below the bridge }\end{array}$ & $\begin{array}{l}42.5542 \mathrm{~N} \\
19.1059 \mathrm{E}\end{array}$ \\
\hline \multirow[t]{2}{*}{$\begin{array}{l}\text { Torrenticola } \\
\text { brevirostris }\end{array}$} & $\begin{array}{l}\frac{42 .}{\mathrm{M} 19} \quad 29 \mathrm{~A} \quad 5 \quad \mathrm{G} 6 \\
\end{array}$ & DNAEC073-20 & BOLD:AED9586 & $\begin{array}{l}\text { Danilovgrad, Zeta } \\
\text { River at Spuž }\end{array}$ & $\begin{array}{l}42.5113 \mathrm{~N} \\
19.1982 \mathrm{E}\end{array}$ \\
\hline & CCDB 38363 C12 & SEPTA036-21 & & $\begin{array}{l}\text { Podgorica, Morača } \\
\text { River in Podgorica }\end{array}$ & $\begin{array}{l}42.4368 \mathrm{~N} \\
19.2559 \mathrm{E}\end{array}$ \\
\hline \multirow[t]{2}{*}{ Torrenticola dudichi } & $\underline{\text { CCDB38233 D11 }}$ & DCCDB047-21 & BOLD:AED7520 & $\begin{array}{l}\text { Mojkovac, Bistrica } \\
\text { stream }\end{array}$ & $\begin{array}{l}42.9871 \mathrm{~N} \\
19.4338 \mathrm{E}\end{array}$ \\
\hline & $\begin{array}{llll}\frac{43 .}{\mathrm{M} 19} & 16 \mathrm{~A} & 4 & \mathrm{G} 7 \\
\end{array}$ & DNAEC074-20 & & $\begin{array}{l}\text { Kolašin, Biogradska } \\
\text { rijeka stream }\end{array}$ & $\begin{array}{l}42.8968 \mathrm{~N} \\
19.6047 \mathrm{E}\end{array}$ \\
\hline
\end{tabular}




\begin{tabular}{|c|c|c|c|c|c|}
\hline Taxa & Voucher Code & $\begin{array}{l}\text { BOLD } \\
\text { Process } \\
\text { ID }\end{array}$ & BIN & Locality & Coordinates \\
\hline \multirow[t]{3}{*}{ Torrenticola laskai } & CCDB-3867-G06 & DNCBD078-20 & \multirow[t]{2}{*}{ BOLD:AEF5471 } & $\begin{array}{l}\text { Podgorica, Cijevna } \\
\text { River at Dinoša }\end{array}$ & $\begin{array}{l}42.4057 \mathrm{~N} \\
19.3569 \mathrm{E}\end{array}$ \\
\hline & CCDB-3867-B10 & DNCBD022-20 & & $\begin{array}{l}\text { Kolašin, Tara River } \\
\text { near Mateševo }\end{array}$ & $\begin{array}{l}42.7898 \mathrm{~N}, \\
19.5374 \mathrm{E}\end{array}$ \\
\hline & CCDB-3867-E11 & DNCBD059-20 & BOLD:AED2306 & $\begin{array}{l}\text { Bar, Međurječka } \\
\text { rijeka stream }\end{array}$ & $\begin{array}{l}42.0363 \mathrm{~N} \\
19.2179 \mathrm{E}\end{array}$ \\
\hline Torrenticola lukai & CCDB $38361 \mathrm{C} 12$ & DCDDJ036-21 & BOLD:ACH9685 & $\begin{array}{l}\text { Kolašin, Bistrica } \\
\text { stream at Crkvine }\end{array}$ & $\begin{array}{l}42.8054 \mathrm{~N} \\
19.4456 \mathrm{E}\end{array}$ \\
\hline \multirow[t]{10}{*}{$\begin{array}{l}\text { Torrenticola } \\
\text { meridionalis }\end{array}$} & CCDB 38361 D02 & $\underline{\text { DCDDJ038-21 }}$ & \multirow[t]{6}{*}{ BOLD:AED7519 } & $\begin{array}{l}\text { Kolašin, Bistrica } \\
\text { stream at Crkvine }\end{array}$ & $\begin{array}{l}42.8054 \mathrm{~N} \\
19.4456 \mathrm{E}\end{array}$ \\
\hline & $\underline{\text { CCDB-3867-G02 }}$ & DNCBD074-20 & & $\begin{array}{l}\text { Bar, Orahovštica } \\
\text { River }\end{array}$ & $\begin{array}{l}42.2476 \mathrm{~N} \\
19.0798 \mathrm{E}\end{array}$ \\
\hline & CCDB-3867-G01 & DNCBD073-20 & & $\begin{array}{l}\text { Bar, Orahovštica } \\
\text { River }\end{array}$ & $\begin{array}{l}42.2476 \mathrm{~N} \\
19.0798 \mathrm{E}\end{array}$ \\
\hline & CCDB-3867-B09 & DNCBD021-20 & & $\begin{array}{l}\text { Kolašin, River } \\
\text { Drcka near } \\
\text { Mateševo }\end{array}$ & $\begin{array}{l}42.7619 \mathrm{~N} \\
19.5549 \mathrm{E}\end{array}$ \\
\hline & CCDB-3867-E01 & DNCBD049-20 & & $\begin{array}{l}\text { Bar, Rikavac stream } \\
\text { above Old Bar }\end{array}$ & $\begin{array}{l}42.1001 \mathrm{~N} \\
19.1432 \mathrm{E}\end{array}$ \\
\hline & CCDB-3867-E03 & DNCBD051-20 & & $\begin{array}{l}\text { Bar, Rikavac stream } \\
\text { above Old Bar }\end{array}$ & $\begin{array}{l}42.1001 \mathrm{~N} \\
19.1432 \mathrm{E}\end{array}$ \\
\hline & $\underline{\mathrm{CCDB}} 38361 \mathrm{D} 01$ & DCDDJ037-21 & \multirow[t]{4}{*}{ BOLD:AEI3402 } & $\begin{array}{l}\text { Kolašin, Bistrica } \\
\text { stream at Crkvine }\end{array}$ & $\begin{array}{l}42.8054 \mathrm{~N} \\
19.4456 \mathrm{E}\end{array}$ \\
\hline & $\underline{\mathrm{CCDB}} 38361 \mathrm{~B} 08$ & DCDDJ020-21 & & $\begin{array}{l}\text { Kolašin, Bistrica } \\
\text { stream at Crkvine }\end{array}$ & $\begin{array}{l}42.8054 \mathrm{~N} \\
19.4456 \mathrm{E}\end{array}$ \\
\hline & CCDB38233 B10 & DCCDB022-21 & & $\begin{array}{l}\text { Kolašin, Kolašinska } \\
\text { rijeka stream }\end{array}$ & $\begin{array}{l}42.8391 \mathrm{~N} \\
19.5749 \mathrm{E}\end{array}$ \\
\hline & CCDB38233 D12 & DCCDB048-21 & & $\begin{array}{l}\text { Mojkovac, Bistrica } \\
\text { stream }\end{array}$ & $\begin{array}{l}42.9871 \mathrm{~N} \\
19.4338 \mathrm{E}\end{array}$ \\
\hline Torrenticola similis & $\underline{\mathrm{CCDB} 38361 \mathrm{~B} 09}$ & DCDDJ021-21 & BOLD:AEK9661 & $\begin{array}{l}\text { Kolašin, Bistrica } \\
\text { stream at Crkvine }\end{array}$ & $\begin{array}{l}42.8054 \mathrm{~N} \\
19.4456 \mathrm{E}\end{array}$ \\
\hline \multirow[t]{2}{*}{ Torrenticola barsica } & CCDB-3867-E09 & DNCBD057-20 & \multirow[t]{2}{*}{ BOLD:AEF1219 } & $\begin{array}{l}\text { Bar, Međurječka } \\
\text { rijeka stream }\end{array}$ & $\begin{array}{l}42.0363 \mathrm{~N} \\
19.2179 \mathrm{E}\end{array}$ \\
\hline & CCDB-3867-F04 & DNCBD064-20 & & $\begin{array}{l}\text { Bar, Međurječka } \\
\text { rijeka stream }\end{array}$ & $\begin{array}{l}42.0363 \mathrm{~N} \\
19.2179 \mathrm{E}\end{array}$ \\
\hline Torrenticola elliptica & $\underline{\text { CDB38233 B11 }}$ & DCCDB023-21 & BOLD:AEI9183 & $\begin{array}{l}\text { Kolašin, Kolašinska } \\
\text { rijeka stream }\end{array}$ & $\begin{array}{l}42.8391 \mathrm{~N} \\
19.5749 \mathrm{E}\end{array}$ \\
\hline \multirow[t]{3}{*}{ Torrenticola ungeri } & $\begin{array}{l}\frac{19 .}{M 19} \quad 24 \quad 6 \quad \text { E10 } \\
\end{array}$ & DNAEC057-20 & \multirow[t]{3}{*}{ BOLD:AED2307 } & $\begin{array}{l}\text { Bar, Međurječka } \\
\text { rijeka stream }\end{array}$ & $\begin{array}{l}42.0226 \mathrm{~N} \\
19.22 \mathrm{E}\end{array}$ \\
\hline & $\begin{array}{l}\frac{20 .}{M 19} \quad 24 \quad 6 \quad E 11 \\
\end{array}$ & DNAEC058-20 & & $\begin{array}{l}\text { Bar, Međurječka } \\
\text { rijeka stream }\end{array}$ & $\begin{array}{l}42.0226 \mathrm{~N} \\
19.22 \mathrm{E}\end{array}$ \\
\hline & CCDB-3867-D08 & DNCBD044-20 & & $\begin{array}{l}\text { Bar, Rikavac stream } \\
\text { above Old Bar }\end{array}$ & $\begin{array}{l}42.1001 \mathrm{~N} \\
19.1432 \mathrm{E}\end{array}$ \\
\hline
\end{tabular}




\begin{tabular}{|c|c|c|c|c|c|}
\hline \multirow[t]{2}{*}{ Taxa } & Voucher Code & $\begin{array}{l}\text { BOLD } \\
\text { Process } \\
\text { ID }\end{array}$ & BIN & Locality & Coordinates \\
\hline & CCDB-3867-G07 & DNCBD079-20 & & $\begin{array}{l}\text { Podgorica, Cijevna } \\
\text { River at Dinoša }\end{array}$ & $\begin{array}{l}42.4057 \mathrm{~N} \\
19.3569 \mathrm{E}\end{array}$ \\
\hline \multirow[t]{2}{*}{$\begin{array}{l}\text { Pseudotorrenticola } \\
\text { rhynchota }\end{array}$} & $\underline{\text { CCDB-3867-B02 }}$ & DNCBD014-20 & \multirow[t]{2}{*}{ BOLD:AEF1632 } & $\begin{array}{l}\text { Bar, stream in } \\
\text { Godinje Village }\end{array}$ & $\begin{array}{l}42.2206 \mathrm{~N} \\
19.1118 \mathrm{E}\end{array}$ \\
\hline & CCDB-3867-B03 & DNCBD015-20 & & $\begin{array}{l}\text { Bar, stream in } \\
\text { Godinje Village }\end{array}$ & $\begin{array}{l}42.2206 \mathrm{~N} \\
19.1118 \mathrm{E}\end{array}$ \\
\hline \multicolumn{6}{|l|}{ Limnesiidae } \\
\hline \multirow[t]{2}{*}{ Limnesia undulata } & $\underline{\text { CCDB-3867-C05 }}$ & DNCBD029-20 & \multirow[t]{2}{*}{ BOLD:AAX5286 } & Tuzi, Vitoja, pools & $\begin{array}{l}42.324 \mathrm{~N} \\
19.3637 \mathrm{E}\end{array}$ \\
\hline & $\underline{\text { CCDB } 38363 \mathrm{CO} 3}$ & $\underline{\text { SEPTA027-21 }}$ & & $\begin{array}{l}\text { Tuzi, Skadar Lake } \\
\text { at Podhum }\end{array}$ & $\begin{array}{l}42.3139 \mathrm{~N}, \\
19.3534 \mathrm{E}\end{array}$ \\
\hline \multicolumn{6}{|l|}{ Hygrobatidae } \\
\hline Atractides fluviatilis & CCDB-3867-G10 & $\underline{\text { DNCBD082-20 }}$ & BOLD:AEF1143 & $\begin{array}{l}\text { Podgorica, Cijevna } \\
\text { River at Dinoša }\end{array}$ & $\begin{array}{l}42.4057 \mathrm{~N} \\
19.3569 \mathrm{E}\end{array}$ \\
\hline \multirow[t]{2}{*}{ Atractides fissus } & CCDB38233 B03 & $\underline{\text { DCCDB015-21 }}$ & \multirow[t]{2}{*}{ BOLD:AEI1811 } & $\begin{array}{l}\text { Kolašin, Kolašinska } \\
\text { rijeka stream }\end{array}$ & $\begin{array}{l}42.8391 \mathrm{~N} \\
19.5749 \mathrm{E}\end{array}$ \\
\hline & $\underline{\text { CCDB38233 D10 }}$ & $\underline{\text { DCCDB046-21 }}$ & & $\begin{array}{l}\text { Mojkovac, Bistrica } \\
\text { stream }\end{array}$ & $\begin{array}{l}42.9871 \mathrm{~N} \\
19.4338 \mathrm{E}\end{array}$ \\
\hline Atractides anae & 1. CG2020 8 B3 & DNAEC014-20 & BOLD:AED1201 & $\begin{array}{l}\text { Berane, spring nr. } \\
\text { Mon. Djurdjevi } \\
\text { Stupovi }\end{array}$ & $\begin{array}{l}42.8527 \mathrm{~N} \\
19.862 \mathrm{E}\end{array}$ \\
\hline $\begin{array}{l}\text { Atractides } \\
\text { inflatipalpis }\end{array}$ & 29. M19 $24 \quad 4 \quad$ F7 & DNAEC064-20 & BOLD:AED3549 & $\begin{array}{l}\text { Bar, Međurječka } \\
\text { rijeka stream }\end{array}$ & $\begin{array}{l}42.0226 \mathrm{~N}, \\
19.22 \mathrm{E}\end{array}$ \\
\hline Atractides inflatipes & $\underline{\text { CCDB-3867-G03 }}$ & DNCBD075-20 & BOLD:AEF1144 & $\begin{array}{l}\text { Bar, Orahovštica } \\
\text { stream }\end{array}$ & $\begin{array}{l}42.2476 \mathrm{~N} \\
19.0798 \mathrm{E}\end{array}$ \\
\hline \multirow[t]{2}{*}{ Atractides fonticolus } & CCDB38233 B09 & DCCDB021-21 & \multirow[t]{2}{*}{ BOLD:AEI8720 } & $\begin{array}{l}\text { Podgorica, Pričelje, } \\
\text { spring Studenac }\end{array}$ & $\begin{array}{l}42.4835 \mathrm{~N} \\
19.2429 \mathrm{E}\end{array}$ \\
\hline & $\underline{\text { CCDB38233 B08 }}$ & $\underline{\text { DCCDB020-21 }}$ & & $\begin{array}{l}\text { Podgorica, Pričelje, } \\
\text { spring Studenac }\end{array}$ & $\begin{array}{l}42.4835 \mathrm{~N} \\
19.2429 \mathrm{E}\end{array}$ \\
\hline \multirow[t]{2}{*}{$\begin{array}{l}\text { Atractides } \\
\text { gibberipalpis }\end{array}$} & $\underline{\text { CCDB } 38361 \mathrm{C} 07}$ & $\underline{\text { DCDDJ031-21 }}$ & BOLD:AEK7766 & $\begin{array}{l}\text { Mojkovac, Bistrica } \\
\text { stream }\end{array}$ & $\begin{array}{l}42.8054 \mathrm{~N} \\
19.4456 \mathrm{E}\end{array}$ \\
\hline & CCDB38233 B02 & DCCDB014-21 & BOLD:AEI3946 & $\begin{array}{l}\text { Kolašin, Kolašinska } \\
\text { rijeka stream }\end{array}$ & $\begin{array}{l}42.8391 \mathrm{~N} \\
19.5749 \mathrm{E}\end{array}$ \\
\hline Atractides inflatus & 14. M19 $12 \quad 4$ E5 & DNAEC052-20 & BOLD:ACB4677 & $\begin{array}{l}\text { Budva, Lastva } \\
\text { Grbaljska, first } \\
\text { order stream }\end{array}$ & $\begin{array}{l}42.3103 \mathrm{~N} \\
18.8138 \mathrm{E}\end{array}$ \\
\hline \multirow[t]{3}{*}{ Atractides nodipalpis } & CCDB-3867-F07 & DNCBD067-20 & \multirow[t]{2}{*}{ BOLD:ACR0209 } & $\begin{array}{l}\text { Danilovgrad, spring } \\
\text { below the bridge }\end{array}$ & $\begin{array}{l}42.5542 \mathrm{~N} \\
19.1059 \mathrm{E}\end{array}$ \\
\hline & $\begin{array}{l}\frac{41 .}{\mathrm{M} 19} \quad 29 \mathrm{~A} \quad 1 \quad \mathrm{G} 5 \\
\end{array}$ & DNAEC072-20 & & $\begin{array}{l}\text { Danilovgrad, Zeta } \\
\text { River at Spuž }\end{array}$ & $\begin{array}{l}42.5113 \mathrm{~N} \\
19.1982 \mathrm{E}\end{array}$ \\
\hline & CCDB-3867-F05 & DNCBD065-20 & BOLD:AED3547 & $\begin{array}{l}\text { Danilovgrad, spring } \\
\text { below the bridge }\end{array}$ & $\begin{array}{l}42.5542 \mathrm{~N} \\
19.1059 \mathrm{E}\end{array}$ \\
\hline
\end{tabular}




\begin{tabular}{|c|c|c|c|c|c|}
\hline Taxa & Voucher Code & $\begin{array}{l}\text { BOLD } \\
\text { Process } \\
\text { ID }\end{array}$ & BIN & Locality & Coordinates \\
\hline & $\begin{array}{l}\frac{40 .}{M 19} \quad 29 A \quad 1 \quad G 4 \\
\end{array}$ & DNAEC071-20 & & $\begin{array}{l}\text { Danilovgrad, Zeta } \\
\text { River at Spuž }\end{array}$ & $\begin{array}{l}42.5113 \mathrm{~N} \\
19.1982 \mathrm{E}\end{array}$ \\
\hline \multirow[t]{10}{*}{ Atractides pennatus } & CCDB-3867-F11 & DNCBD071-20 & \multirow[t]{10}{*}{ BOLD:ADF7007 } & $\begin{array}{l}\text { Bar, Orahovštica } \\
\text { stream }\end{array}$ & $\begin{array}{l}42.2476 \mathrm{~N} \\
19.0798 \mathrm{E}\end{array}$ \\
\hline & CCDB-38679-A09 & DNCBD009-20 & & $\begin{array}{l}\text { Bar, Poseljani, } \\
\text { Poseljanski stream }\end{array}$ & $\begin{array}{l}42.3057 \mathrm{~N} \\
19.0557 \mathrm{E}\end{array}$ \\
\hline & $\begin{array}{l}\frac{25 .}{C G} 2020 \quad 9 \quad C 6 \\
\end{array}$ & DNAEC028-20 & & $\begin{array}{l}\text { Podgorica, Mareza } \\
\text { spring }\end{array}$ & $\begin{array}{l}42.4801 \mathrm{~N} \\
19.1821 \mathrm{E}\end{array}$ \\
\hline & $\begin{array}{l}23 . \\
\text { CG2020 9 C5 }\end{array}$ & DNAEC027-20 & & $\begin{array}{l}\text { Podgorica, Mareza } \\
\text { spring }\end{array}$ & $\begin{array}{l}42.4801 \mathrm{~N} \\
19.1821 \mathrm{E}\end{array}$ \\
\hline & 3. CG2020 2 B4 & DNAEC015-20 & & $\begin{array}{l}\text { Žabljak, Sedlo, } \\
\text { Studenac spring }\end{array}$ & $\begin{array}{l}43.0973 \mathrm{~N} \\
19.0702 \mathrm{E}\end{array}$ \\
\hline & $\begin{array}{l}\frac{32 .}{M 19} \quad 23 \quad 1 \quad F 10 \\
\end{array}$ & DNAEC067-20 & & $\begin{array}{l}\text { Nikšić, Vidrovan, } \\
\text { Vukovo Vrelo spring }\end{array}$ & $\begin{array}{l}42.8575 \mathrm{~N} \\
18.9414 \mathrm{E}\end{array}$ \\
\hline & 31. M19 $23 \quad 1 \quad F 9$ & DNAEC066-20 & & $\begin{array}{l}\text { Nikšić, Vidrovan, } \\
\text { Vukovo Vrelo spring }\end{array}$ & $\begin{array}{l}42.8575 \mathrm{~N} \\
18.9414 \mathrm{E}\end{array}$ \\
\hline & 4. M19 $22 \quad 1 \mathrm{D} 8$ & DNAEC042-20 & & $\begin{array}{l}\text { Nikšić, spring in } \\
\text { Miločani Village }\end{array}$ & $\begin{array}{l}42.8265 \mathrm{~N} \\
18.9018 \mathrm{E}\end{array}$ \\
\hline & $\underline{\mathrm{CCDB}} 38363 \mathrm{C} 01$ & SEPTA025-21 & & $\begin{array}{l}\text { Budva, spring } \\
\text { Smokov Vijenac }\end{array}$ & $\begin{array}{l}42.2346 \mathrm{~N} \\
18.907 \mathrm{E}\end{array}$ \\
\hline & $\underline{\text { CCDB } 38363 \text { B12 }}$ & SEPTA024-21 & & $\begin{array}{l}\text { Budva, spring } \\
\text { Smokov Vijenac }\end{array}$ & $\begin{array}{l}42.2346 \mathrm{~N} \\
18.907 \mathrm{E}\end{array}$ \\
\hline \multirow[t]{5}{*}{ Atractides robustus } & $\underline{\text { CCDB-3867-D12 }}$ & DNCBD048-20 & \multirow[t]{5}{*}{ BOLD:ADZ9348 } & $\begin{array}{l}\text { Bar, Rikavac stream } \\
\text { above Old Bar }\end{array}$ & $\begin{array}{l}42.1001 \mathrm{~N} \\
19.1432 \mathrm{E}\end{array}$ \\
\hline & CCDB-3867-D11 & DNCBD047-20 & & $\begin{array}{l}\text { Bar, Rikavac stream } \\
\text { above Old Bar }\end{array}$ & $\begin{array}{l}42.1001 \mathrm{~N} \\
19.1432 \mathrm{E}\end{array}$ \\
\hline & CCDB-3867-D10 & DNCBD046-20 & & $\begin{array}{l}\text { Bar, Rikavac stream } \\
\text { above Old Bar }\end{array}$ & $\begin{array}{l}42.1001 \mathrm{~N} \\
19.1432 \mathrm{E}\end{array}$ \\
\hline & $\underline{\mathrm{CCDB}} 38361 \mathrm{H} 02$ & DCDDJ086-21 & & $\begin{array}{l}\text { Kolašin, spring nr. } \\
\text { Monastir Morača }\end{array}$ & $\begin{array}{l}42.7668 \mathrm{~N} \\
19.3906 \mathrm{E}\end{array}$ \\
\hline & CCDB38233 B01 & DCCDB013-21 & & $\begin{array}{l}\text { Kolašin, Kolašinska } \\
\text { Rijeka stream }\end{array}$ & $\begin{array}{l}42.8391 \mathrm{~N} \\
19.5749 \mathrm{E}\end{array}$ \\
\hline Atractides latipes & $\begin{array}{l}\frac{18 .}{\mathrm{M} 19} \text { 08B } 7 \quad \text { E9 } \\
\end{array}$ & DNAEC056-20 & BOLD:AED4000 & $\begin{array}{l}\text { Podgorica, River } \\
\text { Cijevna at Trgaja }\end{array}$ & $\begin{array}{l}42.3964 \mathrm{~N} \\
19.3798 \mathrm{E}\end{array}$ \\
\hline \multirow[t]{4}{*}{ Atractides stankovici } & CCDB38233 C08 & $\underline{\mathrm{DCCDB} 032-21}$ & \multirow[t]{4}{*}{ BOLD:AED3550 } & $\begin{array}{l}\text { Dnilovgrad, River } \\
\text { Zeta near Slap }\end{array}$ & $\begin{array}{l}42.6001 \mathrm{~N} \\
19.0656 \mathrm{E}\end{array}$ \\
\hline & CCDB38233 C07 & DCCDB031-21 & & $\begin{array}{l}\text { Danilovgrad, River } \\
\text { Zeta near Slap }\end{array}$ & $\begin{array}{l}42.6001 \mathrm{~N} \\
19.0656 \mathrm{E}\end{array}$ \\
\hline & $\frac{13 . \mathrm{CG} 2020 \_4}{\underline{\mathrm{B} 10}}$ & DNAEC020-20 & & $\begin{array}{l}\text { Podgorica, Mareza } \\
\text { canal }\end{array}$ & $\begin{array}{l}42.479 \mathrm{~N} \\
19.1813 \mathrm{E}\end{array}$ \\
\hline & $\frac{14 . \mathrm{CG} 20204}{\underline{\mathrm{B} 11}}$ & DNAEC021-20 & & $\begin{array}{l}\text { Podgorica, Mareza } \\
\text { canal }\end{array}$ & $\begin{array}{l}42.479 \mathrm{~N} \\
19.1813 \mathrm{E}\end{array}$ \\
\hline
\end{tabular}




\begin{tabular}{|c|c|c|c|c|c|}
\hline Taxa & Voucher Code & $\begin{array}{l}\text { BOLD } \\
\text { Process } \\
\text { ID }\end{array}$ & BIN & Locality & Coordinates \\
\hline \multirow[t]{3}{*}{ Hygrobates calliger } & $\underline{\text { CCDB } 38361 \mathrm{C} 06}$ & $\underline{\text { DCDDJ030-21 }}$ & BOLD:AEL5782 & $\begin{array}{l}\text { Kolašin, Crkvine, } \\
\text { Bistrica stream }\end{array}$ & $\begin{array}{l}42.8054 \mathrm{~N} \\
19.4456 \mathrm{E}\end{array}$ \\
\hline & $\underline{\text { CCDB-38679-A04 }}$ & $\underline{\text { DNCBD004-20 }}$ & \multirow[t]{2}{*}{ BOLD:AEF4261 } & $\begin{array}{l}\text { Bar, Poseljanski } \\
\text { stream at Poseljani }\end{array}$ & $\begin{array}{l}42.3095 \mathrm{~N} \\
19.0518 \mathrm{E}\end{array}$ \\
\hline & $\underline{\text { CCDB-38679-A03 }}$ & DNCBD003-20 & & $\begin{array}{l}\text { Bar, Poseljanski } \\
\text { stream at Poseljani }\end{array}$ & $\begin{array}{l}42.3095 \mathrm{~N} \\
19.0518 \mathrm{E}\end{array}$ \\
\hline Hygrobates foreli & $\underline{\text { Hyd MN VP6 }}$ & DNAEC080-20 & BOLD:AEE3281 & $\begin{array}{l}\text { Žabljak, Mlinski } \\
\text { potok stream }\end{array}$ & $\begin{array}{l}43.1494 \mathrm{~N} \\
19.0898 \mathrm{E}\end{array}$ \\
\hline Hygrobates lacrima & 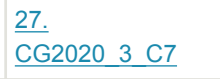 & DNAEC029-20 & BOLD:AED2490 & $\begin{array}{l}\text { Kolašin, Tara River } \\
\text { near Mateševo }\end{array}$ & $\begin{array}{l}42.7897 \mathrm{~N} \\
19.5383 \mathrm{E}\end{array}$ \\
\hline $\begin{array}{l}\text { Hygrobates } \\
\text { limnocrenicus }\end{array}$ & 13. M19 205 E4 & $\underline{\text { DNAEC051-20 }}$ & BOLD:AED2489 & $\begin{array}{l}\text { Podgorica, Mareza } \\
\text { canal }\end{array}$ & $\begin{array}{l}42.479 \mathrm{~N} \\
19.1813 \mathrm{E}\end{array}$ \\
\hline \multirow[t]{4}{*}{$\begin{array}{l}\text { Hygrobates } \\
\text { longipalpis }\end{array}$} & CCDB-3867-C07 & DNCBD031-20 & \multirow[t]{4}{*}{ BOLD:ACR9783 } & Tuzi, Vitoja, pool & $\begin{array}{l}42.324 \mathrm{~N} \\
19.3637 \mathrm{E}\end{array}$ \\
\hline & CCDB-3867-C09 & DNCBD033-20 & & Tuzi, Vitoja, pool & $\begin{array}{l}42.324 \mathrm{~N} \\
19.3637 \mathrm{E}\end{array}$ \\
\hline & $\underline{\text { CCDB-38679-A10 }}$ & $\underline{\text { DNCBD010-20 }}$ & & $\begin{array}{l}\text { Bar, Poseljani, } \\
\text { Poseljanski stream }\end{array}$ & $\begin{array}{l}42.3057 \mathrm{~N} \\
19.0557 \mathrm{E}\end{array}$ \\
\hline & $\underline{\text { CCDB } 38363 \mathrm{CO} 4}$ & SEPTA028-21 & & $\begin{array}{l}\text { Tuzi, Skadar Lake } \\
\text { at Podhum }\end{array}$ & $\begin{array}{l}42.3139 \mathrm{~N} \\
19.3534 \mathrm{E}\end{array}$ \\
\hline \multirow[t]{4}{*}{$\begin{array}{l}\text { Hygrobates } \\
\text { mediterraneus }\end{array}$} & 7. M19 $24 \quad 2$ D11 & DNAEC046-20 & \multirow[t]{4}{*}{ BOLD:AED2190 } & $\begin{array}{l}\text { Bar, Medjurječka } \\
\text { rijeka stream }\end{array}$ & $\begin{array}{l}42.0226 \mathrm{~N}, \\
19.22 \mathrm{E}\end{array}$ \\
\hline & 8. M19 $24 \quad 2 \quad$ D12 & $\underline{\text { DNAEC0 } 47-20}$ & & $\begin{array}{l}\text { Bar, Medjurječka } \\
\text { rijeka stream }\end{array}$ & $\begin{array}{l}42.0226 \mathrm{~N}, \\
19.22 \mathrm{E}\end{array}$ \\
\hline & 36. M19 $24 \quad 1 \quad G 1$ & DNAEC070-20 & & $\begin{array}{l}\text { Bar, Medjurječka } \\
\text { rijeka stream }\end{array}$ & $\begin{array}{l}42.0226 \mathrm{~N}, \\
19.22 \mathrm{E}\end{array}$ \\
\hline & CCDB-3867-F01 & DNCBD061-20 & & $\begin{array}{l}\text { Bar, Medjurječka } \\
\text { rijeka stream }\end{array}$ & $\begin{array}{l}42.0363 \mathrm{~N} \\
19.2179 \mathrm{E}\end{array}$ \\
\hline \multirow[t]{4}{*}{$\begin{array}{l}\text { Hygrobates } \\
\text { norvegicus }\end{array}$} & $\underline{\text { Hyd MN VP3 }}$ & DNAEC077-20 & \multirow[t]{4}{*}{ BOLD:ACH7323 } & $\begin{array}{l}\text { Šavnik, spring } \\
\text { Kikov izvor near } \\
\text { Boan }\end{array}$ & $\begin{array}{l}42.9465 \mathrm{~N} \\
19.1893 \mathrm{E}\end{array}$ \\
\hline & CCDB $38361 \mathrm{~A} 01$ & DCDDJ001-21 & & $\begin{array}{l}\text { Kolašin, Lalevića } \\
\text { Dolovi, spring \#1 }\end{array}$ & $\begin{array}{l}42.899 \mathrm{~N} \\
19.631 \mathrm{E}\end{array}$ \\
\hline & CCDB $38361 \mathrm{~A} 02$ & DCDDJ002-21 & & $\begin{array}{l}\text { Kolašin, Lalevića } \\
\text { Dolovi, spring \#1 }\end{array}$ & $\begin{array}{l}42.899 \mathrm{~N} \\
19.631 \mathrm{E}\end{array}$ \\
\hline & $\underline{\text { CCDB } 38361 \mathrm{~A} 07}$ & DCDDJ007-21 & & $\begin{array}{l}\text { Kolašin, Lalevića } \\
\text { Dolovi, spring \#1 }\end{array}$ & $\begin{array}{l}42.899 \mathrm{~N} \\
19.631 \mathrm{E}\end{array}$ \\
\hline \multicolumn{6}{|l|}{ Unionicolidae } \\
\hline Neumania imitata & $\frac{15 .}{M 19}$ 29C 2 E6 & DNAEC053-20 & BOLD:AED4073 & $\begin{array}{l}\text { Danilovgrad, River } \\
\text { Zeta at Spuž }\end{array}$ & $\begin{array}{l}42.5113 \mathrm{~N} \\
19.1982 \mathrm{E}\end{array}$ \\
\hline Neumania limosa & CCDB-3867-C10 & DNCBD034-20 & BOLD:AEF5902 & Tuzi, Vitoja, pool & $\begin{array}{l}42.324 \mathrm{~N} \\
19.3637 \mathrm{E}\end{array}$ \\
\hline
\end{tabular}




\begin{tabular}{|c|c|c|c|c|c|}
\hline Taxa & Voucher Code & $\begin{array}{l}\text { BOLD } \\
\text { Process } \\
\text { ID }\end{array}$ & BIN & Locality & Coordinates \\
\hline & CCDB-3867-C01 & DNCBD025-20 & & Tuzi, Vitoja, pool & $\begin{array}{l}42.324 \mathrm{~N} \\
19.3637 \mathrm{E}\end{array}$ \\
\hline & CCDB38233 G06 & DCCDB078-21 & & Tuzi, Vitoja, pool & $\begin{array}{l}42.324 \mathrm{~N} \\
19.3637 \mathrm{E}\end{array}$ \\
\hline \multirow[t]{5}{*}{ Unionicola minor } & $\underline{\text { CCDB-3867-G12 }}$ & $\underline{\text { DNCBD084-20 }}$ & \multirow[t]{4}{*}{ BOLD:AEF4865 } & Ulcinj, Šasko Lake & $\begin{array}{l}41.9768 \mathrm{~N} \\
19.3389 \mathrm{E}\end{array}$ \\
\hline & CCDB $38361 \mathrm{C} 02$ & DCDDJ026-21 & & Ulcinj, Šasko Lake & $\begin{array}{l}41.9768 \mathrm{~N} \\
19.3389 \mathrm{E}\end{array}$ \\
\hline & $\underline{\text { CCDB } 38361 \mathrm{C} 05}$ & DCDDJ029-21 & & Ulcinj, Šasko Lake & $\begin{array}{l}41.9768 \mathrm{~N} \\
19.3389 \mathrm{E}\end{array}$ \\
\hline & $\underline{\text { CCDB } 38363 \text { B09 }}$ & $\underline{\text { SEPTA021-21 }}$ & & Tuzi, Vitoja, pool & $\begin{array}{l}42.324 \mathrm{~N} \\
19.3637 \mathrm{E}\end{array}$ \\
\hline & CCDB $38361 \mathrm{C} 04$ & DCDDJ028-21 & BOLD:AAU0335 & Ulcinj, Šasko Lake & $\begin{array}{l}41.9768 \mathrm{~N} \\
19.3389 \mathrm{E}\end{array}$ \\
\hline $\begin{array}{l}\text { Unionicola } \\
\text { ypsilophora }\end{array}$ & $\underline{\text { CCDB } 38363 \text { D04 }}$ & SEPTA040-21 & & $\begin{array}{l}\text { Cetinje, River } \\
\text { Cnojevica ( } \\
\text { Anodonta } \\
\text { exulcerata) }\end{array}$ & $\begin{array}{l}42.3546 \mathrm{~N} \\
19.0286 \mathrm{E}\end{array}$ \\
\hline \multirow[t]{5}{*}{ Piona damkoehleri } & $\underline{\text { CCDB } 38361 \mathrm{~B} 03}$ & DCDDJ015-21 & \multirow[t]{4}{*}{ BOLD:AEK5107 } & $\begin{array}{l}\text { Danilovgrad, } \\
\text { Moromiš pond }\end{array}$ & $\begin{array}{l}42.5322 \mathrm{~N} \\
19.1993 \mathrm{E}\end{array}$ \\
\hline & CCDB 38361 B04 & DCDDJ016-21 & & $\begin{array}{l}\text { Danilovgrad, } \\
\text { Moromiš pond }\end{array}$ & $\begin{array}{l}42.5322 \mathrm{~N} \\
19.1993 \mathrm{E}\end{array}$ \\
\hline & $\underline{\text { CCDB } 38361 \text { B05 }}$ & DCDDJ017-21 & & $\begin{array}{l}\text { Danilovgrad, } \\
\text { Moromiš pond }\end{array}$ & $\begin{array}{l}42.5322 \mathrm{~N} \\
19.1993 \mathrm{E}\end{array}$ \\
\hline & $\underline{\text { CCDB } 38361 \text { B06 }}$ & $\underline{\text { DCDDJ018-21 }}$ & & $\begin{array}{l}\text { Danilovgrad, } \\
\text { Moromiš pond }\end{array}$ & $\begin{array}{l}42.5322 \mathrm{~N} \\
19.1993 \mathrm{E}\end{array}$ \\
\hline & $\underline{\text { CCDB } 38361 \mathrm{~B} 07}$ & DCDDJ019-21 & & $\begin{array}{l}\text { Danilovgrad, } \\
\text { Moromiš pond }\end{array}$ & $\begin{array}{l}42.5322 \mathrm{~N} \\
19.1993 \mathrm{E}\end{array}$ \\
\hline Piona laminata & CCDB $38361 \mathrm{~A} 12$ & DCDDJ012-21 & BOLD:AEL3248 & $\begin{array}{l}\text { Danilovgrad, } \\
\text { Moromiš pond }\end{array}$ & $\begin{array}{l}42.5322 \mathrm{~N} \\
19.1993 \mathrm{E}\end{array}$ \\
\hline \multirow[t]{3}{*}{ Piona disparilis } & $\underline{\text { Hyd MN VP12 }}$ & DNAEC085-20 & \multirow[t]{2}{*}{ BOLD:AEE3977 } & $\begin{array}{l}\text { Šavnik, spring of } \\
\text { Bukovica stream, } \\
\text { pool }\end{array}$ & $\begin{array}{l}43.0589 \mathrm{~N} \\
19.1103 \mathrm{E}\end{array}$ \\
\hline & $\underline{\text { CCDB } 38363 \mathrm{~A} 08}$ & SEPTA008-21 & & $\begin{array}{l}\text { Nikšić, Vukovo } \\
\text { Vrelo spring, pool }\end{array}$ & $\begin{array}{l}42.8577 \mathrm{~N} \\
18.9416 \mathrm{E}\end{array}$ \\
\hline & CCDB 38363 A09 & SEPTA009-21 & & $\begin{array}{l}\text { Nikšić, Vukovo } \\
\text { Vrelo spring, pool }\end{array}$ & $\begin{array}{l}42.8577 \mathrm{~N} \\
18.9416 \mathrm{E}\end{array}$ \\
\hline Typhis torris & $\underline{\text { CCDB-3867-C08 }}$ & DNCBD032-20 & BOLD:AEF2208 & Tuzi, Vitoja, pool & $\begin{array}{l}42.324 \mathrm{~N} \\
19.3637 \mathrm{E}\end{array}$ \\
\hline \multirow[t]{2}{*}{ Typhis ornatus } & CCDB 38361 B01 & DCDDJ013-21 & \multirow[t]{2}{*}{ BOLD:ACS0401 } & $\begin{array}{l}\text { Danilovgrad, } \\
\text { Moromiš pond }\end{array}$ & $\begin{array}{l}42.5322 \mathrm{~N} \\
19.1993 \mathrm{E}\end{array}$ \\
\hline & CCDB 38361 B02 & DCDDJ014-21 & & $\begin{array}{l}\text { Danilovgrad, } \\
\text { Moromiš pond }\end{array}$ & $\begin{array}{l}42.5322 \mathrm{~N} \\
19.1993 \mathrm{E}\end{array}$ \\
\hline
\end{tabular}




\begin{tabular}{|c|c|c|c|c|c|}
\hline Taxa & Voucher Code & $\begin{array}{l}\text { BOLD } \\
\text { Process } \\
\text { ID }\end{array}$ & BIN & Locality & Coordinates \\
\hline \multicolumn{6}{|l|}{ Wettinidae } \\
\hline Wettina lacustris & 30. M19 $20 \quad 4 \quad F 8$ & DNAEC065-20 & BOLD:ADL2726 & $\begin{array}{l}\text { Podgorica, Mareza } \\
\text { canal }\end{array}$ & $\begin{array}{l}42.479 \mathrm{~N} \\
19.1813 \mathrm{E}\end{array}$ \\
\hline \multicolumn{6}{|l|}{ Mideopsidae } \\
\hline $\begin{array}{l}\text { Mideopsis } \\
\text { milankovici }\end{array}$ & $\begin{array}{l}\frac{22 .}{M 19} \quad 24 \quad 2 \quad E 12 \\
\underline{y}\end{array}$ & DNAEC059-20 & BOLD:AED2191 & $\begin{array}{l}\text { Bar, Medjurječka } \\
\text { rijeka stream }\end{array}$ & $\begin{array}{l}42.0226 \mathrm{~N}, \\
19.22 \mathrm{E}\end{array}$ \\
\hline \multirow[t]{5}{*}{$\begin{array}{l}\text { Mideopsis } \\
\text { roztoczensis }\end{array}$} & $\underline{\text { CCDB-38679-A02 }}$ & DNCBD002-20 & \multirow[t]{5}{*}{ BOLD:ACI1492 } & $\begin{array}{l}\text { Cetinje, Poseljanski } \\
\text { stream }\end{array}$ & $\begin{array}{l}42.3095 \mathrm{~N} \\
19.0518 \mathrm{E}\end{array}$ \\
\hline & CCDB-3867-G05 & DNCBD077-20 & & $\begin{array}{l}\text { Podgorica, Cijevna } \\
\text { River at Dinoša }\end{array}$ & $\begin{array}{l}42.4057 \mathrm{~N} \\
19.3569 \mathrm{E}\end{array}$ \\
\hline & $\underline{\text { CCDB38233 C12 }}$ & DCCDB036-21 & & $\begin{array}{l}\text { Danilovgrad, Zeta } \\
\text { River at Spuž }\end{array}$ & $\begin{array}{l}42.5112 \mathrm{~N} \\
19.1991 \mathrm{E}\end{array}$ \\
\hline & $\underline{\text { CCDB38233 C11 }}$ & $\underline{\text { DCCDB035-21 }}$ & & $\begin{array}{l}\text { Danilovgrad, Zeta } \\
\text { River at Spuž }\end{array}$ & $\begin{array}{l}42.5112 \mathrm{~N} \\
19.1991 \mathrm{E}\end{array}$ \\
\hline & $\underline{\text { CCDB } 38363 \text { D07 }}$ & $\underline{\text { SEPTA043-21 }}$ & & $\begin{array}{l}\text { Danilovgrad, Zeta } \\
\text { River at Vranjske } \\
\text { Njive }\end{array}$ & $\begin{array}{l}42.4683 \mathrm{~N} \\
19.2579 \mathrm{E}\end{array}$ \\
\hline \multicolumn{6}{|c|}{ Athienemanniidae } \\
\hline $\begin{array}{l}\text { Mundamella } \\
\text { germanica }\end{array}$ & 1. KIA 20B D6 & DNAEC041-20 & BOLD:AED6269 & $\begin{array}{l}\text { Danilovgrad, Spuž, } \\
\text { spring near Zeta } \\
\text { River }\end{array}$ & $\begin{array}{l}42.5113 \mathrm{~N} \\
19.1982 \mathrm{E}\end{array}$ \\
\hline \multicolumn{6}{|l|}{ Aturidae } \\
\hline \multirow[t]{3}{*}{$\begin{array}{l}\text { Hexaxonopsis } \\
\text { serrata }\end{array}$} & $\underline{\text { CCDB } 38363 \text { B01 }}$ & SEPTA013-21 & & $\begin{array}{l}\text { Bar, Skadar Lake at } \\
\text { Murići }\end{array}$ & $\begin{array}{l}42.1637 \mathrm{~N} \\
19.2214 \mathrm{E}\end{array}$ \\
\hline & CCDB 38363 B02 & $\underline{\text { SEPTA014-21 }}$ & & $\begin{array}{l}\text { Bar, Skadar Lake at } \\
\text { Murići }\end{array}$ & $\begin{array}{l}42.1637 \mathrm{~N} \\
19.2214 \mathrm{E}\end{array}$ \\
\hline & CCDB 38363 B03 & $\underline{\text { SEPTA015-21 }}$ & & $\begin{array}{l}\text { Bar, Skadar Lake at } \\
\text { Murići }\end{array}$ & $\begin{array}{l}42.1637 \mathrm{~N} \\
19.2214 \mathrm{E}\end{array}$ \\
\hline $\begin{array}{l}\text { Parabrachypoda } \\
\text { montii }\end{array}$ & 5. M19 $20 \quad 6 \quad$ D9 & DNAEC044-20 & BOLD:AED5455 & $\begin{array}{l}\text { Podgorica, Mareza } \\
\text { canal }\end{array}$ & $\begin{array}{l}42.479 \mathrm{~N} \\
19.1813 \mathrm{E}\end{array}$ \\
\hline \multirow[t]{2}{*}{$\begin{array}{l}\text { Woolastokia } \\
\text { rotundifrons }\end{array}$} & 10. M19 $27 \quad 2$ E1 & DNAEC048-20 & BOLD:AEE0289 & $\begin{array}{l}\text { Šavnik, Tušina } \\
\text { River at Boan }\end{array}$ & $\begin{array}{l}42.9432 \mathrm{~N} \\
19.205 \mathrm{E}\end{array}$ \\
\hline & 11. M19 $27 \quad 2$ E2 & DNAEC049-20 & & $\begin{array}{l}\text { Šavnik, Tušina } \\
\text { River at Boan }\end{array}$ & $\begin{array}{l}42.9432 \mathrm{~N} \\
19.205 \mathrm{E}\end{array}$ \\
\hline \multicolumn{6}{|l|}{ Arrenuridae } \\
\hline $\begin{array}{l}\text { Arrenurus } \\
\text { cylindratus }\end{array}$ & $\begin{array}{llll}\frac{34 .}{M 19} & 20 & 1 & F 12 \\
\end{array}$ & DNAEC069-20 & BOLD:AED6864 & $\begin{array}{l}\text { Podgorica, Mareza } \\
\text { canal }\end{array}$ & $\begin{array}{l}42.479 \mathrm{~N} \\
19.1813 \mathrm{E}\end{array}$ \\
\hline $\begin{array}{l}\text { Arrenurus } \\
\text { refractarioulus }\end{array}$ & $\underline{\text { CCDB } 38363 \text { A07 }}$ & $\underline{\text { SEPTA007-21 }}$ & & $\begin{array}{l}\text { Nikšić, Lukavica } \\
\text { Mt., pools }\end{array}$ & $\begin{array}{l}42.8118 \mathrm{~N} \\
19.1872 \mathrm{E}\end{array}$ \\
\hline
\end{tabular}


All obtained BINs were inspected for concordance using BOLD Workbench. The Refined Single Linkage (RESL) algorithm was used to assign water mite barcodes to Operational Taxonomic Units (OTUs).

Sequence comparisons were performed using MUSCLE alignment (Edgar 2004). Intraand interspecific genetic distances were calculated, based on the Kimura 2-parameter model (K2P; Kimura 1980), using MEGA-X, version 10.1 (Kumar et al. 2018). The Neighbour-Joining (NJ) tree (edited in MEGA7, Kumar et al. 2016), based on K2P distances and pairwise deletion of missing data, was used to visualise similarity. The support for tree branches was calculated by the non-parametric bootstrap method (Felsenstein 1985) with 1000 replicates and shown next to the branches.

\section{Results}

DNA barcodes of 233 specimens morphologically assigned to 86 species from 28 genera and 15 families of water mites from Montenegro were newly generated for this study. The specimens were collected through the "DNA-Eco" (DNA barcode reference library as a tool for sustainable management of freshwater ecosystems in the highly threatened Lake Skadar Basin) project. The current study develops the first COI barcode reference library of water mites for Montenegro with the focus on Skadar/Shkodra Lake catchment area.

Fragment lengths of the analysed DNA barcode fragments ranged from 201 to 658 (mean: 636.2) base pairs, including no stop codons, insertions or deletions. The DNA barcode region was characterised by a high AT-content: the mean sequence compositions were $A=30.82 \pm 0.1252 \%, C=20.39 \pm 0.1222 \%, G=14.91 \pm 0.0709 \%$ and $T=33.88 \pm$ $0.1253 \%$. The obtained results are similar to those found in other arthropod studies (e.g. Raupach et al. 2015).

The families Hygrobatidae Koch, 1842 and Lebertiidae Thor, 1900 are represented by the highest number of sequences (53 and 44, respectively). The opposite, the three families Arrenuridae Thor, 1900, Teutoniidae Koenike, 1910 and Limnesiidae Thor, 1900 are represented each with two sequences and the two familes Athienemanniidae K. Viets, 1922 and Wettinidae Cook, 1956 by the lowest number of sequences (each with one sequence). The most common genus was Lebertia Neuman, 1880, for which 44 barcode sequences (11 species) were generated, followed by Atractides Koch, 1837 (35 barcodes; 13 species), Torrenticola Piersig, 1896 and Sperchon Kramer, 1877 (29 and 26 barcodes, 10 and 8 species, respectively). Six genera were represented by a single specimen. The highest number of barcodes per species was reached for Atractides pennatus (K. Viets, 1922), Sperchon violaceus Walter, 1944 and Torrenticola meridionalis Di Sabatino and Cicolani, 1990 (each with 10 barcodes), followed by Lebertia inaequalis (Koch, 1837) and L. variolata Gerecke, 2009 (each with 8 barcodes) and Sperchon thienemanni Koenike, 1907 (6 barcodes). On the other hand, most species are represented by less than 5 DNA barcodes. Thirty-three species are represented by a single DNA barcode not allowing us to estimate the intraspecific distances. BOLD ID and accession numbers for all specimens included in final dataset are given in Table 1. 
The mean intrageneric K2P distance was $20.2 \pm 0.0 \%$ (range 6.09-42.37\%). The mean intraspecific nucleotide K2P distances were $2.43 \pm 0.01 \%$ (ranging from $0 \%$ to $24.16 \%$ ). The summary statistics showing significant changes of average K2P distances within the different taxonomic levels are given in Table 2.

Table 2.

Summary table of K2P genetic distances within the different taxonomic levels derived from 233 analysed water mite specimens from Montenegro. The list of studied species is provided in Table 1.

Deletion Method: Pairwise Deletion. Alignment: BOLD Aligner (Amino Acid based HMM).

\begin{tabular}{|l|l|l|l|l|l|l|l|}
\hline Label & $\mathbf{n}$ & Taxa & Comparisons & Min Dist. (\%) & Mean Dist. (\%) & Max Dist. (\%) & SE Dist. (\%) \\
\hline Within Species & 200 & 53 & 391 & 0.00 & 2.43 & 24.16 & 0.01 \\
\hline Within Genus & 207 & 14 & 2291 & 6.09 & 20.20 & 42.37 & 0.00 \\
\hline Within Family & 168 & 7 & 1054 & 16.17 & 37.14 & 63.16 & 0.01 \\
\hline
\end{tabular}

The BIN and RESL (OTU) analyses assigned sequences to 98 BINs and 103 OTUs, respectively. Fifty BINs (159 records) were concordant $(51 \%)$ and 48 BINs were represented by a single sequence $(49 \%)$. At the time of publication of the dataset, fifty-five $(56.1 \%)$ of these BINs (with 102 sequences) included sequences only from Montenegro, while the remaining BINs included sequences also from other countries.

Most of the morphologically-identified species show an intraspecific variation of less than $2 \%$. However, the 14 taxa listed in Table 3 showed a maximum interspecific divergence larger than $2 \%$, resulting in these species in BOLD being spread over more than one BIN. Two species, Lebertia glabra Thor, 1897 and L. inaequalis appeared each with 3 BINs and twelve species, i.e. Lebertia maculosa Koenike, 1902, L. porosa Thor, 1900, Sperchon brevirostris Koenike, 1895, S. clupeifer Piersig, 1896, Sperchonopsis verrucosa (Protz, 1896), Monatractides madritensis (K. Viets, 1930), Torrenticola meridionalis, T. laskai Di Sabatino, 2009, Atractides gibberipalpis Piersig, 1898, A. nodipalpis Thor, 1899, Hygrobates calliger Piersig, 1896 and Unionicola minor (Soar, 1900), each with 2 BINs (Table 3). In total, unique BINs were revealed for 72 species $(83.7 \%)$, two BINs for 12 species (14.0\%) and three BINs for two species (2.3\%).

The NJ analyses, based on K2P distances, revealed non-overlapping clusters with bootstrap support values $>95 \%$ for 50 species $(58 \%)$ with more than one analysed specimen indicating a high congruence between BINs affiliation and morphological species identification. Moreover, specimens showing high intraspecific distances are also clearly separated into different clades. A more detailed topology of all analysed specimens is presented in the supporting information (Suppl. material 2).

\section{Discussion}

This study provides $\mathrm{COI}$ barcodes for 233 specimens representing 86 morphologically identified species of water mites from Montenegro. These represent $42.8 \%$ of Montenegrin 
water mite fauna, based on Pešić et al. (2018) and papers published thereafter (Pešić et al. 2019c, Pešić et al. 2020a, Pešić et al. 2020c, Pešić et al. 2020d, Pešić and Smit 2020). BOLD and RESL (OTU) analyses revealed 98 BINs and 103 OTUs, respectively, highlighting the high molecular diversity of the water mite fauna of Montenegro.

Table 3.

Species with intraspecific (ISD) maximum pairwise distances $>2.2 \%$ (p-dist.). Divergence values were calculated for all studied sequences, using the Nearest Neighbour Summary, implemented in the Barcode Gap Analysis tool provided by the Barcode of Life Data System (BOLD). BINs are based on the barcode analysis from 15 November 2021. Country codes (alpha-2 code): BG = Bulgaria, $\mathrm{CH}=$ Switzerland, $\mathrm{DE}=$ Germany, $\mathrm{ES}=$ Spain, $\mathrm{FR}=$ France, $\mathrm{GB}=$ United Kingdom, $\mathrm{GL}=$ Greenland $\mathrm{IT}=$ Italy, NO = Norway, $\mathrm{NL}=$ Netherlands, $\mathrm{ME}=$ Montenegro, $\mathrm{MK}=$ North Macedonia, $\mathrm{PL}=$ Poland, $\mathrm{RO}=$ Romania, $\mathrm{RS}=$ Serbia, $\mathrm{RU}=$ Russia, $\mathrm{SK}=$ Slovakia $. n=\mathrm{BIN}$ member count.

\begin{tabular}{|c|c|c|c|c|c|c|c|c|}
\hline No. & Species & BIN & $n$ & MeanISD & MaxISD & Country & $\begin{array}{l}\text { Nearest BIN/ } \\
\text { Species }\end{array}$ & $\begin{array}{l}\text { Distance } \\
\text { to NN }\end{array}$ \\
\hline \multirow[t]{3}{*}{1.} & Lebertia glabra & BOLD:ACR9598 & 2 & 0.8 & 0.8 & $\mathrm{ME}, \mathrm{NL}$ & BOLD:ACS0595 & 12.52 \\
\hline & Lebertia glabra & BOLD:ACS0595 & 20 & 0.64 & 1.36 & $\begin{array}{l}\text { NL, BG, ME, } \\
\text { MK, IT, PL, SK }\end{array}$ & BOLD:AEJ3212 & 2.88 \\
\hline & Lebertia glabra & BOLD:AEI925 & 3 & 0.64 & 0.96 & ME & BOLD:ACO2179 & 12.02 \\
\hline \multirow[t]{3}{*}{2.} & $\begin{array}{l}\text { Lebertia } \\
\text { inaequalis }\end{array}$ & BOLD:AEF5913 & 1 & $\mathrm{~N} / \mathrm{A}$ & $\mathrm{N} / \mathrm{A}$ & ME & BOLD:ADF6223 & 2.78 \\
\hline & $\begin{array}{l}\text { Lebertia } \\
\text { inaequalis }\end{array}$ & BOLD:ADF6223 & 18 & 0.18 & 0.34 & $\mathrm{NL}, \mathrm{PL}, \mathrm{ME}$ & $\underline{\text { BOLD:AEF5913 }}$ & 2.78 \\
\hline & $\begin{array}{l}\text { Lebertia } \\
\text { inaequalis }\end{array}$ & BOLD:AEF2742 & 1 & $\mathrm{~N} / \mathrm{A}$ & N/A & ME & BOLD:AEB4193 & 6.96 \\
\hline \multirow[t]{2}{*}{3.} & $\begin{array}{l}\text { Lebertia } \\
\text { maculosa }\end{array}$ & BOLD:AED 9718 & 3 & 1.27 & 1.6 & ME, MK & BOLD:AED9197 & 2.76 \\
\hline & $\begin{array}{l}\text { Lebertia } \\
\text { maculosa }\end{array}$ & BOLD:AED9197 & 2 & 0.16 & 0.16 & ME & BOLD:AED9718 & 2.76 \\
\hline \multirow[t]{2}{*}{4.} & Lebertia porosa & BOLD:ACS0974 & 133 & 0.81 & 2.37 & $\begin{array}{l}\text { NL, FR, ME, } \\
\text { DE, GB, BG, } \\
\text { IT, PL, SK, ES, } \\
\text { CH }\end{array}$ & BOLD:AED4662 & 3.89 \\
\hline & Lebertia porosa & BOLD:AED4662 & 12 & 0.14 & 0.85 & ME & BOLD:ACS0974 & 3.89 \\
\hline \multirow[t]{2}{*}{5.} & $\begin{array}{l}\text { Sperchon } \\
\text { brevirostris }\end{array}$ & BOLD:AED3857 & 3 & 0.32 & 0.48 & ME, MK & BOLD:AEK3053 & 2.72 \\
\hline & $\begin{array}{l}\text { Sperchon } \\
\text { brevirostris }\end{array}$ & BOLD:ACP6107 & 28 & 0.55 & 3.12 & $\mathrm{NO}, \mathrm{DE}, \mathrm{ME}$ & BOLD:AED3857 & 7.53 \\
\hline \multirow[t]{2}{*}{6.} & $\begin{array}{l}\text { Sperchon } \\
\text { clupeifer }\end{array}$ & BOLD:ACS1100 & 11 & 1.68 & 3.47 & $\begin{array}{l}\text { NL, DE, NO, } \\
\text { MK, ME, RU }\end{array}$ & BOLD:AEE4061 & 8.7 \\
\hline & $\begin{array}{l}\text { Sperchon } \\
\text { clupeifer }\end{array}$ & BOLD:AEE4061 & 1 & $\mathrm{~N} / \mathrm{A}$ & N/A & ME & BOLD:ACS1100 & 8.7 \\
\hline 7. & $\begin{array}{l}\text { Sperchonopsis } \\
\text { verrucosa }\end{array}$ & BOLD:AEK8297 & 1 & $\mathrm{~N} / \mathrm{A}$ & N/A & ME, RO & BOLD:ACS0908 & 4.83 \\
\hline
\end{tabular}




\begin{tabular}{|c|c|c|c|c|c|c|c|c|}
\hline No. & Species & BIN & $n$ & MeanISD & MaxISD & Country & $\begin{array}{l}\text { Nearest BIN/ } \\
\text { Species }\end{array}$ & $\begin{array}{l}\text { Distance } \\
\text { to NN }\end{array}$ \\
\hline & $\begin{array}{l}\text { Sperchonopsis } \\
\text { verrucosa }\end{array}$ & BOLD:ACS9705 & 9 & 0.29 & 0.97 & NO, IT, ME & BOLD:ADU8190 & 9.83 \\
\hline \multirow[t]{2}{*}{8.} & $\begin{array}{l}\text { Monatractides } \\
\text { madritensis }\end{array}$ & BOLD:AED3803 & 2 & 0.16 & 0.16 & ME & BOLD:AEL3852 & 1.44 \\
\hline & $\begin{array}{l}\text { Monatractides } \\
\text { madritensis }\end{array}$ & BOLD:AEL3852 & 2 & 0.64 & 0.64 & $M E, S R$ & BOLD:AED3803 & 1.44 \\
\hline \multirow[t]{2}{*}{9.} & $\begin{array}{l}\text { Torrenticola } \\
\text { meridionalis }\end{array}$ & BOLD:AED7519 & 8 & 1.46 & 2.25 & ME, MK & BOLD:AEI3402 & 6.57 \\
\hline & $\begin{array}{l}\text { Torrenticola } \\
\text { meridionalis }\end{array}$ & BOLD:AEI3402 & 4 & 1.42 & 2.09 & ME & BOLD:AEK9662 & 6.25 \\
\hline \multirow[t]{2}{*}{10.} & $\begin{array}{l}\text { Torrenticola } \\
\text { laskai }\end{array}$ & BOLD:AEF5471 & 2 & 0.32 & 0.32 & ME & BOLD:AED2306 & 2.17 \\
\hline & $\begin{array}{l}\text { Torrenticola } \\
\text { laskai }\end{array}$ & BOLD:AED2306 & 4 & 0.82 & 1.34 & RS, ME, RO & BOLD:AEF5471 & 2.17 \\
\hline \multirow[t]{2}{*}{11.} & $\begin{array}{l}\text { Atractides } \\
\text { gibberipalpis }\end{array}$ & BOLD:AEK7766 & 1 & N/A & N/A & ME & BOLD:AEI3946 & 4.81 \\
\hline & $\begin{array}{l}\text { Atractides } \\
\text { gibberipalpis }\end{array}$ & BOLD:AEI3946 & 1 & N/A & N/A & ME & BOLD:AEK7766 & 4.81 \\
\hline \multirow[t]{2}{*}{12.} & $\begin{array}{l}\text { Atractides } \\
\text { nodipalpis }\end{array}$ & BOLD:ACR0209 & 41 & 0.59 & 3.05 & $\begin{array}{l}\text { NO, NL, GL, } \\
\text { DE, ME, RS }\end{array}$ & BOLD:AED3548 & 13.3 \\
\hline & $\begin{array}{l}\text { Atractides } \\
\text { nodipalpis }\end{array}$ & BOLD:AED3547 & 2 & 0 & 0 & ME & BOLD:AAM4306 & 13.3 \\
\hline \multirow[t]{2}{*}{13.} & $\begin{array}{l}\text { Hygrobates } \\
\text { calliger }\end{array}$ & BOLD:AEF4261 & 2 & 1.2 & 1.2 & NO, ME & BOLD:AEK4720 & 16.18 \\
\hline & $\begin{array}{l}\text { Hygrobates } \\
\text { calliger }\end{array}$ & BOLD:AEL5782 & 2 & 1.03 & 1.03 & $\mathrm{DE}, \mathrm{ME}$ & BOLD:AEK4720 & 14.61 \\
\hline \multirow[t]{2}{*}{14.} & Unionicola minor & BOLD:AEF4865 & 3 & 0.59 & 0.7 & ME & BOLD:ACI7165 & 17.02 \\
\hline & Unionicola minor & BOLD:AAU0335 & 7 & 0.09 & 0.32 & $\mathrm{NO}, \mathrm{NL}, \mathrm{ME}$ & BOLD:ACH 3803 & 16.03 \\
\hline
\end{tabular}

Of the 86 species recorded in this study, 79 species were previously reported for Montenegro. DNA barcoding confirmed the presence of four species new for Montenegro, i.e. Lebertia reticulata (Koenike, 1919), Atractides inflatipalpis K.Viets, 1950, A. latipes (Szalay, 1935) and Parabrachypoda montii (Maglio, 1924). Three species, i.e. Protzia octopora Lundblad, 154, Piona laminata (Thor, 1901) and Unionicola ypsilophora (Bonz, 1783) are recorded for the first time for the Balkan Peninsula. Specimens of the latter species were found between the gill blades of mussels Anodonta exulcerata Clesin, 1876, whose identification was confirmed by molecular data.

Moreover, species identification, based on molecular data conducted during this project, extended the list of Montenegrin water mites by description of several species new for science, i.e. Atractides anae Pešić, 2020, Hygrobates lacrima Pešić, 2020, H. limnocrenicus Pešić, 2020, H mediterraneus Pešić, 2020 and Mideopsis milankovici Pešić and Smit, 2020 (Pešić et al. 2020a, Pešić et al. 2020c, Pešić et al. 2020d, Pešić and Smit 
2020). All of these studies highlighted the importance of an integrated approach that combines the morphology-based taxonomy and DNA barcodes.

Our study confirmed efficiency of DNA barcoding as a tool for the identification of water mites. In particular, 72 of the 86 morphologically-identified species exactly matched the BINs defined from BOLD. This result coincides with high identification efficiency rates through the BOLD Best Close Match analysis. Nevertheless, our data revealed also 14 species listed in Table 3 that showed high intraspecific distances (> 2.2\%) suggesting possible cryptic and/or pseudocryptic diversification. Most of these possible cryptic and/or pseudocryptic species, as seen in Table 3, appear to be hidden within common species.

Three species, i.e. Lebertia maculosa, Monatractides madritensis and Torrenticola laskai appeared each with 2 BINs in our dataset. The intraspecific maximum distances between BINs within each of these species were below $3 \%$ (Suppl. material 1). On the other hand, the intraspecific maximum distances between BINs within each of the other eleven species in the dataset were greater than $5 \%$ (Suppl. material 1).

Lebertia glabra, a species widely distributed in West Palaearctic (Di Sabatino et al. 2010) appeared in our dataset with 3 BINs. The first cluster (BIN:ACR9598) includes two specimens from Montenegro and The Netherlands; the second cluster (BIN:ACS0595) was more represented in BOLD and includes specimens from different parts of Europe - from The Netherlands and Poland to Montenegro, Italy and Macedonia. The third cluster (BOLD:AEI925) contained only specimens from Montenegro. The intraspecific K2P distances between all clusters ranged from 14.3 to $17.7 \%$ (Suppl. material 1).

Lebertia inaequalis, a species reported from the extended parts of the Palaearctic (Gerecke 2009, Di Sabatino et al. 2010), appeared in our dataset with 3 BINs, two of which each include only one specimen from Montenegro (BIN:AEF5913 and BIN:AEF2742, respectively). The third cluster (BIN:ADF6223), based on available records from BOLD, appears to be more widespread and contained specimens from The Netherlands, Poland and Montenegro. Intraspecific K2P distances between the latter cluster and BIN:AEF5913 was only $0.1 \%$, while the distance from the second cluster (BIN:AEF2742) from Montenegro was rather large (17.3\%; Suppl. material 1) highlighting the necessity of additional comprehensive morphological and molecular analysis.

Lebertia porosa, a eurytopic and eurythermous species, often reported from standing waters and pools of streams across the Holarctic (Gerecke 2009, Di Sabatino et al. 2010), is currently in the process of being revised (R. Gerecke, pers. communication) using DNA barcodes. Stur (2017) showed that 18 specimens of L. porosa from Norway comprise 7 BINs with a mean intraspecific $p$-distance of $11.7 \%$ and maximum up to $18.5 \%$. In our dataset, specimens, morphologically assigned to Lebertia porosa, were presented with two clusters. Based on the available records from BOLD, the first cluster (BIN:ACS0974) appeared to be well represented in the BOLD database with 133 records from different parts of Europe; the second cluster (BIN:AED4662) contained specimens only from Montenegro. In our study, specimens of the latter BIN were collected in large limnocrenic springs, such as Mareza and Vitoja, while specimens from the first cluster (BIN:ACS0974) 
were sampled in the lower reaches. The intraspecific K2P distance between these two $L$. porosa clusters in our dataset was estimated at 5.5\% (Suppl. material 1).

Sperchon brevirostris, a species inhabiting low-and middle order streams in the study area (Pešić et al. 2010, Pešić et al. 2018), was represented in our material by two clusters. Based on the available records from BOLD, the first cluster (BIN:ACP6107) includes specimens from Norway, Germany and one specimen from Montenegro, while the second cluster (BOLD:AED3857) contained three specimens from Montenegro and North Macedonia. The K2P distance between these two clusters was 8.1\% (Suppl. material 1). Similarly, S. clupeifer, a species frequently reported from Western Palaearctic (Di Sabatino et al. 2010), appeared with two clusters in our dataset. The first cluster (BIN:ACS1100) is well represented in BOLD and includes specimens from different part of Europe, while the second cluster (BIN:AEE4061) contained a single specimen from Montenegro. The intraspecific K2P distance between these two clusters in our dataset was estimated at $8.3 \%$ (Suppl. material 1 ).

Sperchonopsis verrucosa, a species often reported from the Holarctic Region (Gerecke et al. 2016), was represented in our study with two clusters. The first cluster (BIN:ACS9705) was more represented in BOLD and includes specimens from Norway, Italy and one specimen from Montenegro. The second cluster (BIN:AEK8297) includes two specimens from Montenegro and Romania. The intraspecific K2P distances between these two clusters was $11.2 \%$, indicating the need for additional integrative analysis.

Torrenticola meridionalis, a species originally described from Italy, is widely distributed in Montenegro, inhabiting mainly low order streams (Pešić et al. 2018). It is morphologically closely related to $T$. elliptica which remains distinguishable in the male sex only, based on the stouter genital field. In our COI tree (Suppl. material 2), T. elliptica appeared as a sister clade to the clade that includes two clusters morphologically assigned to $T$. meridionalis (BIN:AEI3402 and BOLD:AED7519, respectively). The intraspecific K2P distances between $T$. elliptica and T. meridionalis clusters ranged from 8.6-9.0\%. On the other hand, the $\mathrm{K} 2 \mathrm{P}$ distance between T. meridionalis clusters in our dataset was estimated at $6.6 \%$.

Atractides nodipalpis, a rhitrobiontic species, is the most frequently reported species of the genus in Europe (Gerecke et al. 2016). In our dataset, sequences of the specimens, morphologically assigned to the latter species, appeared as two clusters. Interestingly, specimens of both clusters were recorded syntopically. The first cluster (BIN:ACR0209) in the BOLD database was represented with 41 specimens from Norway (country of the type locality), The Netherlands, Montenegro and Russia, but also from Greenland. The second cluster (BIN:AED3547) includes two specimens from Montenegro. The intraspecific K2P distance between these two clusters was $18.8 \%$, indicating the need for a comprehensive revision of this species complex.

Hygrobates calliger, a rhitrobiontic species widely distributed in the Palaearctic (Di Sabatino et al. 2010), was represented by two clusters in our dataset, each with two records in the BOLD database. The first cluster (BIN:AEF4261) includes specimens from Norway and Montenegro, while the second cluster (BIN:AEL5782) includes specimens 
from Germany and Montenegro. The intraspecific K2P distance between these two clusters was $20.9 \%$ (Suppl. material 1), suggesting the existence of possible hidden cryptic and/or pseudocryptic species.

The sequences of Atractides gibberipalpis, a rhitrobiontic species often reported from the Palaearctic (Pešić et al. 2021a), in our dataset were assigned to two different barcode clusters, each represented by a single specimen from Montenegro. The intraspecific K2P distance between these two clusters (BIN: BOLD:AEK7766 and BIN: BOLD:AEI3946, respectively) was estimated at $5 \%$ (Suppl. material 1 ).

Unionicola minor, a species widely distributed in Europe (Gerecke et al. 2016), was presented with two clusters in our dataset. Based on available data from BOLD, the first cluster (BIN:AAU0335) includes specimens from Norway and The Netherlands and one specimen from Lake Šasko in Montenegro. The second cluster (BIN:AAU0335) includes specimens only from Montenegro. The intraspecific K2P distances between these two clusters in our dataset was $23.8 \%$ (Suppl. material 1), suggesting the existence of cryptic (or pseudocryptic, see Pešić and Smit (2016) for a discussion about pseudocryptic speciation in water mites) species. Stålstedt et al. (2013) showed that the Swedish population of Unionicola minor consists of at least three cryptic species, emphasising the need for further research of the species in this complex.

Taxonomic studies of the above species were outside the scope of this paper. Further studies with material from a wider geographical area, were needed to clarify taxonomy and elucidate the delimitation of the species in the above complexes. This process should be accompanied by sufficient barcode coverage to allow the detection of phylogeographic patterns and/or even the existence of possible overlooked cryptic species. The build-up of DNA barcode library for water mites of Montenegro represents a long-term task, aimed at improving molecular identification and inclusion of this group in environmental assessment programmes and, on the other hand, to stimulate further biodiversity research of this limnofaunistic group in Montenegro and the Balkans.

\section{Acknowledgements}

The authors are grateful to all those who helped during sample collections in Montenegro. This study is part of the "DNA-Eco" scientific project, supported by a grant of the Montenegrin Ministry of Science. The part of study was supported by Polish National Science Centre, Poland, grant no. 2017/27/N/NZ8/01568.

\section{Ethics and security}

No ethical principles were violated when providing this study. 


\section{Conflicts of interest}

The authors declare no conflict of interests concerning this study.

\section{References}

- $\quad$ Baker R, Pešić V, Gerecke R, Hristovski N, Stojanovski S (2008) A comparative analysis of the water mite fauna (Acari) of three transboundary lakes in the Balkans.

Lauterbornia 62: 45-51.

- Bańkowska A, Kłosowska M, Gadawski P, Michoński G, Grabowski M, Pešić V, Zawal A (2016) Oviposition by selected water mite (Hydrachnidia) species from Lake Skadar and its catchment. Biologia 71 (9): 1027-1033. https://doi.org/10.1515/biolog-2016-0126

- $\quad$ Blattner L, Gerecke R, von Fumetti S (2019) Hidden biodiversity revealed by integrated morphology and genetic species delimitation of spring dwelling water mite species (Acari, Parasitengona: Hydrachnidia). Parasites \& Vectors 12 (492). https://doi.org/ 10.1186/s13071-019-3750-y

- $\quad$ Davids C, Di Sabatino A, Gerecke R, Gledhill T, Smit H, Hammen H (2007) Acari: Hydrachnidia I. In: Gerecke R (Ed), Chelicerata, Acari I. Süßwasserfauna von Mitteleuropa, 7, 2-1. Spektrum Elsevier, München, 241-376 pp.

- Di Sabatino A, Gerecke R, Smit H, Pešić V, Panesar A (2003) Water mites of the family Torrenticolidae (Acari, Actinedida, Hydrachnidia) from the eastern Mediterranean region. Archiv für Hydrobiologie Supplement 139 (3): 1-39.

- Di Sabatino A, Gerecke R, Gledhill T, Smit H (2010) Acari: Hydrachnidia II. In: Gerecke R (Ed.), Chelicerata: Acari II. Süßwasserfauna von Mitteleuropa, Vol. 7, 2-2. Elsevier Spektrum Akademischer Verlag, Heidelberg, 1-234 pp.

- $\quad$ Edgar RC (2004) MUSCLE: multiple sequence alignment with high accuracy and high throughput. Nucleic Acids Research 32 (5): 1792-1797. https://doi.org/10.1093/nar/ gkh340

- Felsenstein J (1985) Confidence limits on phylogenies: An approach using the bootstrap. Evolution 39 (4): 783-791. https://doi.org/10.2307/2408678

- $\quad$ Fisher JR, Fisher D, Skvarla M, Nelson W, Dowling AG (2017) Revision of torrent mites (Parasitengona, Torrenticolidae, Torrenticola) of the United States and Canada: 90 descriptions, molecular phylogenetics, and a key to species. ZooKeys 701: 1-496. https://doi.org/10.3897/zookeys.701.13261

- $\quad$ Gerecke R (2009) Revisional studies on the European species of the water mite genus Lebertia Neuman, 1880 (Acari: Hydrachnidia: Lebertiidae). Abhandlungen der Senckenberg Gesellschaft für Naturforschung, 144 pp.

- Gerecke R, Gledhill T, Pešić V, Smit H (2016) Chelicerata: Acari III. In: Gerecke R (Ed), Süßwasserfauna von Mitteleuropa, Bd. 7/2-3. Springer-Verlag, Berlin, Heidelberg, 429 pp. https://doi.org/10.1007/978-3-8274-2689-5

- Ivanova NV, Grainger CM (2007a) CCDB protocols, COI amplification. Canadian Centre for DNA Barcoding URL: ccdb.ca/site/wp-content/uploads/2016/09/

CCDB Amplification.pdf

- Ivanova NV, Grainger CM (2007b) CCDB protocols, sequencing. Canadian Centre for DNA Barcoding URL: ccdb.ca/site/wp-content/uploads/2016/09/CCDB Sequencing.pdf 
- Ivanova NV, deWaard JR, Hebert PD (2007) CCDB protocols, glass fiber plate DNA extraction. Canadian Centre for DNA Barcoding URL: http://ccdb.ca/site/wp-content/ uploads/2016/09/CCDB DNA Extraction.pdf

- Kimura M (1980) A simple method for estimating evolutionary rates of base substitutions through comparative studies of nucleotide sequences. Journal of Molecular Evolution 16 (2): 111-120. https://doi.org/10.1007/bf01731581

- Kumar S, Stecher G, Tamura K (2016) MEGA7: Molecular Evolutionary Genetics Analysis Version 7.0 for Bigger Datasets. Molecular Biology and Evolution 33 (7): 1870-1874. https://doi.org/10.1093/molbev/msw054

- $\quad$ Kumar S, Stecher G, Li M, Knyaz C, Tamura K (2018) MEGA X: Molecular Evolutionary Genetics Analysis across computing platforms. Molecular Biology and Evolution 35 (6): 1547-1549. https://doi.org/10.1093/molbev/msy096

- Martin P, Dabert M, Dabert J (2010) Molecular evidence for species separation in the water mite Hygrobates nigromaculatus Lebert, 1879 (Acari, Hydrachnidia): evolutionary consequences of the loss of larval parasitism. Aquatic Sciences 72 (3): 347-360.

https://doi.org/10.1007/s00027-010-0135-x

- Musselius AA (1912) Einige Hydracarinen aus Montenegro. Trudy Varšhavskago Obšhčhestva Estestvoispytatelei 23 (3-4): 95-97.

- Pešić V (2001) Stygohydracarus karanovici sp.n., and Atractides inflatipes Lundblad, 1956, two water mites species (Acari: Hydrachnidia) from Montenegro (SE Europe). Zootaxa 17 (1): 1-7. https://doi.org/10.11646/zootaxa.17.1.1

- Pešić V (2002a) Hydrodroma reinhardi sp. n., a new species of water mites (Acari, Actinedida, Hydrodromidae) from the Mediterranean Area. Aquatic Insects 24 (4): 317-323. https://doi.org/10.1076/aqin.24.4.317.8239

- Pešić V (2002b) Water mites (Acari, Actinedida) of the stagnant waters from the Skadar Lake drainage basin (Crna Gora, Yugoslavia. Acta Entomologica

Serbica 5 (1-2): 131-152.

- $\quad$ Pešić V (2002c) Two interesting species of the genus Atractides Koch 1837 (Acari, Actinedida) from Crna Gora (Balkan peninsula). Lauterbornia 44: 65-71.

- Pešić V (2002d) First description of the male of Atractides graecus K. Viets, 1950 (Acari, Actinedida, Hygrobatidae) from Montenegro (Yugoslavia). The Montenegrin Academy of Sciences and Arts, Glasnik of the Section of Natural Sciences 14: 177-182.

- Pešić V (2003a) On some very interesting water mite species (Acari, Actinedida) from Crna Gora (Montenegro), new for the Balkan peninsula and Mediterranean region. Natura Montenegrina 1: 89-98.

- Pešić V (2003b) New records of water mites (Acari, Hydrachnidia) from Yugoslavia. Archives of Biological Sciences, Belgrade 54 (3-4): 25-26. https://doi.org/10.2298/ ABS020425PP

- $\quad$ Pešić V (2003c) New records of water mites (Acari: Hydrachnidia) from running waters from Montenegro and FYR Macedonia (SE Europe). Acta Entomologica Serbica 6 (1-2): 131-128.

- Pešić V, Gerecke R (2003) Water mites of the genera Albaxona, Axonopsis, Barbaxonella and Erebaxonopsis (Acari, Hydrachnidia: Aturidae: Axonopsinae) from Central Europe and Mediterranean area. Archiv für Hydrobiologie Supplement 139 (4): 563-576.

- $\quad$ Pešić V (2004a) Water mites (Acari: Hydrachnidia) of the Biogradska Gora National Park (Serbia and Crna Gora). In: Pešić V, et al. (Ed.) The biodiversity of the Biogradska 
Gora National Park. Monographies, I. Department of Biology, University of Montenegro \& Centre for Biodiversity of Montenegro, Podgorica, 65-86 pp.

- Pešić V (2004b) Some new and rare water mites (Acari: Hydrachnidia) from the Balkan peninsula. Glasnik Republičkog Zavoda za Zaštitu Prirode - Prirodnjačkog muzeja. 27-28. 93-99 pp.

- $\quad$ Pešić V, Smit H, Gerecke R, Di Sabatino A (2010) The water mites (Acari: Hydrachnidia) of the Balkan peninsula, a revised survey with new records and descriptions of five new taxa. Zootaxa 2586: 1-100. https://doi.org/10.11646/zootaxa.2586.1.1

- Pešić V, Valdecasas A, Garcia-Jimenez R (2012) Simultaneous evidence for a new species of Torrenticola Piersig, 1896 (Acari, Hydrachnidia) from Montenegro. Zootaxa 3515: 38-50. https://doi.org/10.11646/zootaxa.3515.1.2

- $\quad$ Pešić V, Smit H (2016) Evidence of cryptic and pseudocryptic speciation in Brachypodopsis baumi species complex (Acari, Hydrachnidia, Aturidae) from Borneo, with description of three new species. Systematic and Applied Acarology 21 (8): 1092-1106. https://doi.org/10.11158/saa.21.8.10

- Pešić V, Asadi M, Cimpean M, Dabert M, Esen Y, Gerecke R, Martin P, Savić A, Smit H, Stur E (2017) Six species in one: evidence of cryptic speciation in the Hygrobates fluviatilis complex (Acariformes, Hydrachnidia, Hygrobatidae). Systematic and Applied Acarology 22 (9): 1327-1377. https://doi.org/10.11158/saa.22.9.4

- Pešić V, Bańkowska A, Goldschmidt T, Grabowski M, Michoński G, Zawal A (2018) Supplement to the checklist of water mites (Acari: Hydrachnidia) from the Balkan peninsula. Zootaxa 4394 (2): 151-184. https://doi.org/10.11646/zootaxa.4394.2.1

- $\quad$ Pešić V, Broda L, Dabert M, Gerecke R, Martin P, Smit H (2019a) Re-established after hundred years: Definition of Hygrobates prosiliens Koenike, 1915, based on molecular and morphological evidence, and redescription of $H$. longipalpis (Hermann, 1804) (Acariformes, Hydrachnidia, Hygrobatidae). Systematic and Applied Acarology 24 (8): 1490-1511. https://doi.org/10.11158/saa.24.8.10

- $\quad$ Pešić V, Dmitrović D, Savić A, Milošević $Đ$, Zawal A, Vukašinović-Pešić $V$, Von Fumetti $S(2019 b)$ Application of macroinvertebrate multimetrics as a measure of the impact of anthropogenic modification of spring habitats. Aquatic Conservation: Marine and Freshwater Ecosystems 29 (3): 341-352. https://doi.org/10.1002/aqc.3021

- Pešić V, Savić A, Jabłońska A, Michoński G, Grabowski M, Bańkowska A, Zawal A (2019c) Environmental factors affecting water mite assemblages along eucrenonhypocrenon gradients in Mediterranean karstic springs. Experimental and Applied Acarology 77 (4): 471-486. https://doi.org/10.1007/s10493-019-00360-w

- $\quad$ Pešić V, Smit H (2020) Mideopsis milankovici sp. nov. a new water mite from Montenegro based on morphological and molecular data (Acariformes, Hydrachnidia, Mideopsidae). Acarologia 60 (3): 566-575. https://doi.org/10.24349/acarologia/ 20204387

- Pešić V, Zawal A, Bańkowska A, Jovanović M, Dabert M (2020a) A new crenobiontic water mite species of the genus Atractides Koch, 1837 from Montenegro and Bulgaria, based on morphological and molecular data (Acariformes, Hydrachnidia, Hygrobatidae). Systematic and Applied Acarology 25 (10): 1889-1900. https://doi.org/10.11158/saa. 25.10 .12

- $\quad$ Pešić V, Saboori A, Jovanović M, Manović A, Bańkowska A, Zawal A (2020b) Torrenticola dowlingi sp. nov. a new water mite from Iran based on morphometrical and 
molecular data (Acariformes, Hydrachnidia, Torrenticolidae). International Journal of Acarology 46 (5): 298-303. https://doi.org/10.1080/01647954.2020.1802513

- Pešić V, Jovanović M, Manović A, Zawal A, Bańkowska A, Broda $Ł$, Martin P, Dabert M (2020c) Two new species from the Hygrobates nigromaculatus-complex (Acariformes, Hydrachnidia, Hygrobatidae), based on morphological and molecular evidence.

Acarologia 60 (4): 753-768. https://doi.org/10.24349/acarologia/20204400

- Pešić V, Jovanović M, Manović A, Zawal A, Bańkowska A, Lyubomirova L, Karaouzas I, Dabert M (2020d) Molecular evidence for two new species of the Hygrobates fluviatilis complex from the Balkan Peninsula (Acariformes, Hydrachnidia, Hygrobatidae).

Systematic and Applied Acarology 25 (9): 1702-1719. https://doi.org/10.11158/saa. 25.9.15

- Pešić V, Smit H, Saboori A (2021a) New records of the water mite genus Atractides Koch, 1837 from Iran (Acari: Hydrachnidia: Hygrobatidae). Ecologica Montenegrina 44: 1-10. https://doi.org/10.37828/em.2021.44.1

- $\quad$ Pešić V, Smit H, Gülle P, Dabert M (2021b) Molecular DNA barcoding of the water mite genus Protzia Persig, 1896 with a description of three new species and the unknown male of $P$. longiacetabulata (Acari, Hydrachnidia). Systematic and Applied Acarology 26 (7): 1213-1228. https://doi.org/10.11158/saa.26.7.3

- Ratnasingham S, Hebert PN (2013) A DNA-based registry for all animal species: The Barcode Index Number (BIN) system. PLOS One 8 (7): 1-16. https://doi.org/10.1371/ journal.pone.0066213

- $\quad$ Raupach M, Barco A, Steinke D, Beermann J, Laakmann S, Mohrbeck I, Neumann H Kihara T, Pointner K, Radulovici A, Segelken-Voigt A, Wesse C, Knebelsberger T (2015) The application of DNA barcodes for the identification of marine crustaceans from the North Sea and adjacent regions. PLOS One 10 (9): e0139421. https://doi.org/10.1371/ journal.pone. 0139421

- Smit H, Pešić V (2004) New records of the families Arrenuridae, Nudomideopsidae and Athienemanniidae (Acari: Hydrachnidia) from Macedonia and Yugoslavia. Acta Entomologica Serbica 7 (1-2): 137-146.

- Smit H (2020) Water mites of the world, with keys to the families, subfamilies genera and subgenera (Acari: Hydrachnidia). Monografieën van de Nederlandse Entomologische Vereniging 12: 1-774.

- $\quad$ Stålstedt J, Bergsten J, Ronquist F (2013) "Forms" of water mites (Acari: Hydrachnidia): intraspecific variation or valid species? Ecology and Evolution 3: 3415-3435. https:// doi.org/10.1002/ece3.704

- $\quad$ Stur E (2017) DNA barcoding of Norwegian water mites. NTNU University Museum URL: https://www.norbol.org/en/dna-barcoding-of-norwegian-water-mites

- Thon K (1903) Über die in Montenegro von Dr. Mrázek gesammelten Hydrachniden. Die Königliche Böhmische Gesellschaft der Wissenschaften, Prag. II. Classe, 1-7 pp.

- Viets K (1936) Hydracarinen aus Jugoslavien (Systematische, ökologische, faunistische und tiergeographische Untersuchungen über die Hydrachnellae und Halacaridae des Süßwassers. Archiv für Hydrobiologie 29: 351-409.

- Weigand H, Beermann A, Čiampor F, Costa F, Csabai Z, Duarte S, Geiger M, Grabowski M, Rimet F, Rulik B, Strand M, Szucsich N, Weigand A, Willassen E, Wyler S, Bouchez A, Borja A, Čiamporová-Zat'ovičová Z, Ferreira S, Dijkstra K, Eisendle U, Freyhof J, Gadawski P, Graf W, Haegerbaeumer A, van der Hoorn B, Japoshvili B, Keresztes L, Keskin E, Leese F, Macher J, Mamos T, Paz G, Pešić V, Pfannkuchen DM, 
Pfannkuchen MA, Price B, Rinkevich B, Teixeira ML, Várbíró G, Ekrem T (2019) DNA barcode reference libraries for the monitoring of aquatic biota in Europe: Gap-analysis and recommendations for future work. Science of The Total Environment 678: 499-524. https://doi.org/10.1016/j.scitotenv.2019.04.247

- Zawal A, Pešić V (2018) The Diversity of Water Mite Assemblages (Acari:

Parasitengona: Hydrachnidia) of Lake Skadar/Shkodra and Its Catchment Area. In: Pešić V, Karaman G, Kostianoy A (Eds) The Skadar/Shkodra Lake Environment. The Handbook of Environmental Chemistry, 80. Springer, Cham, 311-323 pp. https://doi.org/ $10.1007 / 698 \quad 2018257$

- Zawal A, Bańkowska A, Michoński G, Grabowski M, Szlauer-Łukaszewska A, Czernicki T, Stępień E, Płóciennik M, Pešić V (2020) Environmental determinants of water mite (Acari: Hydrachnidia) distribution in the ancient Lake Skadar system. Journal of Great Lakes Research 46 (5): 1090-1098. https://doi.org/10.1016/j.jglr.2019.06.002

\section{Supplementary materials}

\section{Suppl. material 1: Molecular distances doi}

Authors: Vladimir Pešić, Andrzej Zawal, Ana Manović, Aleksandra Bańkowska, Milica Jovanović Data type: Molecular distances

Brief description: Molecular distances, based on the Kimura 2-parameter model of the analysed specimens of water mites from Montenegro. BINs are based on the barcode analysis from 15 November 2021.

Download file $(79.41 \mathrm{~kb})$

\section{Suppl. material 2: Compact Neighbour-Joining tree doi}

Authors: Vladimir Pešić, Andrzej Zawal, Ana Manović, Aleksandra Bańkowska, Milica Jovanović Data type: Neighbour-joining tree

Brief description: Compact Neighbour-Joining tree of all analysed water mite species based on Kimura 2-parameter distances. The tree was edited in MEGA7 (Kumar et al. 2016). Specimens are classified using ID numbers from BOLD and species name. BINs are based on the barcode analysis from 15 November 2021. Numbers next to nodes represent non-parametric bootstrap values $(1,000$ replicates, in \%). The analyses involved all $233 \mathrm{COI}$ nucleotide sequences.

Download file $(62.83 \mathrm{~kb})$ 